ARTICLE

\title{
Structural and molecular basis of ZNRF3/RNF43 transmembrane ubiquitin ligase inhibition by the Wnt agonist R-spondin
}

Matthias Zebisch ${ }^{1}$, Yang $\mathrm{Xu}^{2,3}$, Christos Krastev ${ }^{1}$, Bryan T. MacDonald ${ }^{2}$, Maorong Chen ${ }^{2}$, Robert J.C. Gilbert ${ }^{1}, \mathrm{Xi} \mathrm{He}^{2} \& \mathrm{E}$. Yvonne Jones ${ }^{1}$

The four R-spondin (Rspo) proteins are secreted agonists of Wnt signalling in vertebrates, functioning in embryogenesis and adult stem cell biology. Through ubiquitination and degradation of Wnt receptors, the transmembrane E3 ubiquitin ligase ZNRF3 and related RNF43 antagonize Wnt signalling. Rspo ligands have been reported to inhibit the ligase activity through direct interaction with ZNRF3 and RNF43. Here we report multiple crystal

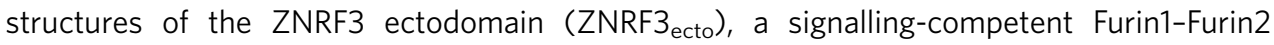
(Fu1-Fu2) fragment of Rspo2 (Rspo2 Fu1-Fu2 $_{2}$, and Rspo2 ${ }_{\mathrm{Fu} 1-\mathrm{Fu} 2}$ in complex with ZNRF3 ${ }_{\text {ector }}$ or RNF43 ecto. A prominent loop in Fu1 clamps into equivalent grooves in the ZNRF3 ecto and RNF43 ecto surface. Rspo binding enhances dimerization of ZNRF3 ecto but not of RNF43 ${ }_{\text {ecto }}$ Comparison of the four Rspo proteins, mutants and chimeras in biophysical and cellular assays shows that their signalling potency depends on their ability to recruit ZNRF3 or RNF43 via Fu1 into a complex with LGR receptors, which interact with Rspo via Fu2.

\footnotetext{
${ }^{1}$ Division of Structural Biology, Wellcome Trust Centre for Human Genetics, University of Oxford, Oxford OX3 7BN, UK. ${ }^{2}$ F.M. Kirby Neurobiology Center, Department of Neurology, Boston Children's Hospital, Harvard Medical School, Boston, Massachusetts 02115, USA. ${ }^{3}$ Key Laboratory for Molecular Enzymology and Engineering of Ministry of Education, College of Life Science, Jilin University, Changchun 130012, China. Correspondence and requests for materials should be addressed to E.Y.J. (email: yvonne@strubi.ox.ac.uk).
} 
$\mathrm{R}$ spo (R-spondin, also roof plate-specific spondin) proteins are evolutionarily conserved from fish to humans and have well-documented roles in a broad range of developmental and physiological processes resulting from enhancement of canonical and non-canonical Wnt signalling ${ }^{1-3}$. Rspol is involved in mammalian sex determination ${ }^{4}$ and is a potent stimulator of epithelial repair in the gastrointestinal tract ${ }^{2,5}$. Rspo 2 has recently been identified as a major determinant of susceptibility to infectious diarrhoea in mice, linking infection and intestinal homoeostasis ${ }^{6}$. Gene fusions involving RSPO2 and RSPO3 have been found in $10 \%$ of primary colon cancers ${ }^{7}$, whereas mutations in RSPO4 underlie inherited anonychia, a disorder in nail development ${ }^{8-10}$. The leucine-rich repeat containing $\mathrm{G}$ protein-coupled receptors 4,5 and 6 (LGR4/5/6) are conserved high-affinity cell surface receptors for Rspo proteins ${ }^{11-15}$; however, the molecular mechanisms by which Rspo proteins function have remained obscure.

Recently published work has indicated that Rspo proteins can exert their potentiating effects on Wnt signalling through direct interaction with the extracellular regions of ZNRF3 or RNF43, ultimately inducing formation of a complex comprising ZNRF3/ RNF43, Rspo and LGR4/5/6 (ref. 16). Similar to the Rspo proteins, ZNRF3 and RNF43 are highly conserved in vertebrates. Loss-offunction mutations of RNF43 in pancreatic cancer have implicated it as a tumour suppressor ${ }^{17}$. ZNRF3 and RNF43 comprise an amino-terminal extracellular region of uncharacterized topology and moderate sequence conservation of $39 \%$ identity between the two proteins, a transmembrane region and a cytoplasmic region that bears the hallmark sequence of a really interesting new gene (RING)-type E3 ubiquitin ligase. Similar to LGR4/5/6 receptors, ZNRF3/RNF43 have been reported to associate in the membrane with the Wnt receptor Frizzled and LRP5/6 coreceptors ${ }^{13,16}$. ZNRF3/RNF43 specifically targets these Wnt receptors for ubiquitination and turnover, hence reducing Wnt signalling responses ${ }^{16,18}$. Direct extracellular interaction with Rspo proteins inhibits ZNRF3/RNF43 activity ${ }^{16}$. These observations have led to the suggestion that Rspo acts to physically bridge between its two receptor types ZNRF3/RNF43 and LGR4/5/6 (ref. 16). Current models suggest that membrane clearance of ZNRF3/ RNF43 through this ternary complex relieves turnover of Wnt receptors and hence enhances Wnt responsiveness.

Here we report a molecular level analysis of the ZNRF3/RNF43 ectodomain structure and its interactions with Rspo proteins. Our study provides mechanistic insight into this key control point in the Wnt signalling pathway.

\section{Results}

Structure determination. Sequence analyses suggest a putative domain structure for the Rspo proteins comprising two furin-like cysteine-rich regions (Fu domains) plus a thrombospondin type 1 repeat domain ${ }^{3}$ (Fig. 1a). Our own and published data point to the involvement of the $\mathrm{Fu}$ domains in the potentiation of canonical Wnt signalling by Rspo proteins ${ }^{1,19-21}$ (Supplementary Fig. S1). We therefore engineered constructs to express the region spanning the two $\mathrm{Fu}$ domains of Rspo 2 proteins from several species. We also generated secreted forms of the corresponding ZNRF3 and RNF43 ectodomains. The Rspo2 $2_{\mathrm{Fu} 1-\mathrm{Fu} 2}$ and respective $\mathrm{ZNRF} 3_{\text {ecto }}$ or RNF43 ${ }_{\text {ecto }}$ molecules migrated together in gel filtration chromatography indicating high-affinity binding (data not shown), substantiating their ligand-receptor relationship. By using a combination of heavy atom and molecular-replacementbased phasing strategies, we determined multiple crystal structures for Xenopus (x) Rspo2 $2_{\mathrm{Fu} 1-\mathrm{Fu} 2}$ (highest resolution 2.2 ${ }$ ), $\mathrm{xZNRF} 3_{\text {ecto }}$ zebrafish (z) ZNRF3 ${ }_{\text {ecto }}$ and mouse (m) ZNRF3 $3_{\text {ecto, }}$ (highest resolutions 2.4, 1.6 and 2.0 , respectively), plus complexes comprising $\mathrm{xZNRF} 3_{\text {ecto }}-\mathrm{xRspo} 2_{\mathrm{Fu} 1-\mathrm{Fu} 2}, \mathrm{mZNRF} 3_{\text {ecto }}-$ $\mathrm{mRspo} 2_{\mathrm{Fu} 1-\mathrm{Fu} 2}, \mathrm{mZNRF} 3_{\text {ecto }}-\mathrm{xRspo} 2_{\mathrm{Fu} 1-\mathrm{Fu} 2}$ and $\mathrm{xRNF} 4 \mathrm{~F} 3_{\text {ecto }}-$ xRspo $_{\text {Fu1-Fu2 }}$ (at 2.1, 2.8, 2.4 and $2.7 \AA$, respectively; see Methods, Table 1 and Supplementary Table S1). In the following sections and figures, the highest resolution structures (Table 1 and Fig. 1) for the apo ligand, apo receptor and ligandreceptor complex will be used unless otherwise stated.

Structure of the ZNRF3 ectodomain. The $\mathrm{ZNRF}_{\text {ecto }}$ crystal structures revealed a distinctive variant of the protease-associated domain topology $y^{22}$. Two $\beta$-sheets (comprising $\beta 2, \beta 1, \beta 7, \beta 3$ and $\beta 4, \beta 5, \beta 6$ strands, respectively; Fig. 1b) splay apart, accommodating an $\alpha$-helix $(\alpha C)$ at the open edge; two additional $\alpha$-helices $(\alpha \mathrm{A}$ and $\alpha \mathrm{B})$ pack against the $\beta 4, \beta 5$ and $\beta 6$ face of this distorted $\beta$-sandwich. A disulphide bridge, conserved across species, links two structurally elaborate loops, $\beta 3-\beta 4$ and $\beta 4-\alpha \mathrm{A}$. The resultant single-domain structure is relatively compact. The crystal structures for apo $x Z N R F 3_{\text {ecto }}$ zZNRF3 $3_{\text {ecto }}$ and $m Z N R F 3_{\text {ecto }}$ showed no major differences in the main chain conformation (Supplementary Fig. S2). Comparisons of ZNRF3 $3_{\text {ecto }}$ structures for proteins crystallized in several different crystal lattices, or for crystals containing multiple copies in the asymmetric unit, consistently highlighted an acidic region (N105-E114; residue numbering is for mouse sequences unless otherwise stated) within the $\beta 3-\beta 4$ loop, the short $\alpha \mathrm{C}-\beta 7$ loop and the extended $\beta 1-\beta 2$ hairpin as flexible elements of the fold (Supplementary Fig. S2). A search of the Protein Data Bank for structures with a similar topology yielded the ectodomain of GRAIL (gene related to anergy in lymphocytes) as the closest match (deposited as an unpublished crystal structure by J.R. Walker and colleagues, Structural Genomics Consortium; Protein Data Bank ID code 3ICU). GRAIL is a single-span transmembrane E3 ubiquitin ligase, which localizes to the endosomal compartment and promotes CD3 ubiquitinylation, acting as an essential regulator of T-cell tolerance ${ }^{23,24}$. The sequence identity between ZNRF3 and GRAIL ectodomains is low ( $13.4 \%$ for 127 residues); however, structural superposition revealed a shared three-dimensional fold consistent with a common evolutionary origin (r.m.s.d. $2.5 \AA$ for 131 equivalent $\mathrm{C} \alpha$ pairs; Supplementary Fig. S3a).

Our crystallographic data provided independent structures for multiple copies of the ZNRF3 ectodomain in eight different crystal forms (Table 1 and Supplementary Table S1). All but two of these crystal structures reveal an extensive interface (average interface area $992 \pm 109 \AA^{2}$; Supplementary Table S2) formed between two $\mathrm{ZNRF}_{\text {ecto }}$ polypeptide chains. This dimer is conserved, and pairwise structural superpositions yielded r.m.s.d. values of $<1.3 \AA$ (for 275 equivalent $\mathrm{C} \alpha$ pairs; Supplementary Tables S3 and S4). The interaction is twofold symmetric; strands $\beta 3$ and $\beta 7$ of the 'subunits' abut face-to-face at the core of the dimer (Fig. $1 \mathrm{c}$ and $2 \mathrm{a}$ ). The $\beta 1-\beta 2$ hairpin forms a second interface by reaching out to embrace helix $\alpha \mathrm{A}$ and the $\beta 3-$ $\beta 4$ loop in the opposing subunit (Fig. 2b). Intriguingly, these structural features interact in a parallel (cis) fashion consistent with ZNRF3 associating as a dimer on the cell surface.

Structure of a signalling-competent fragment of Rspo2. In the crystal structure of the isolated Rspo Fu1-Fu2 $_{2}$ protein (Table 1) the two $\mathrm{Fu}$ domains arrange sequentially to form a ladder-like structure of $\beta$-hairpins (Fig. 1d). Each Fu domain comprises three $\beta$-hairpins rigidified by four disulphide bridges (Fig. 1e), similar to the cysteine-rich regions found in members of the epidermal growth factor receptor family (Supplementary Fig. S3b). The connection between the $\mathrm{Fu}$ domains shows considerable rotational freedom, allowing a $50^{\circ}-60^{\circ}$ variation in the relative interdomain orientation (Supplementary Fig. S4). The $\mathrm{N}$ terminal 


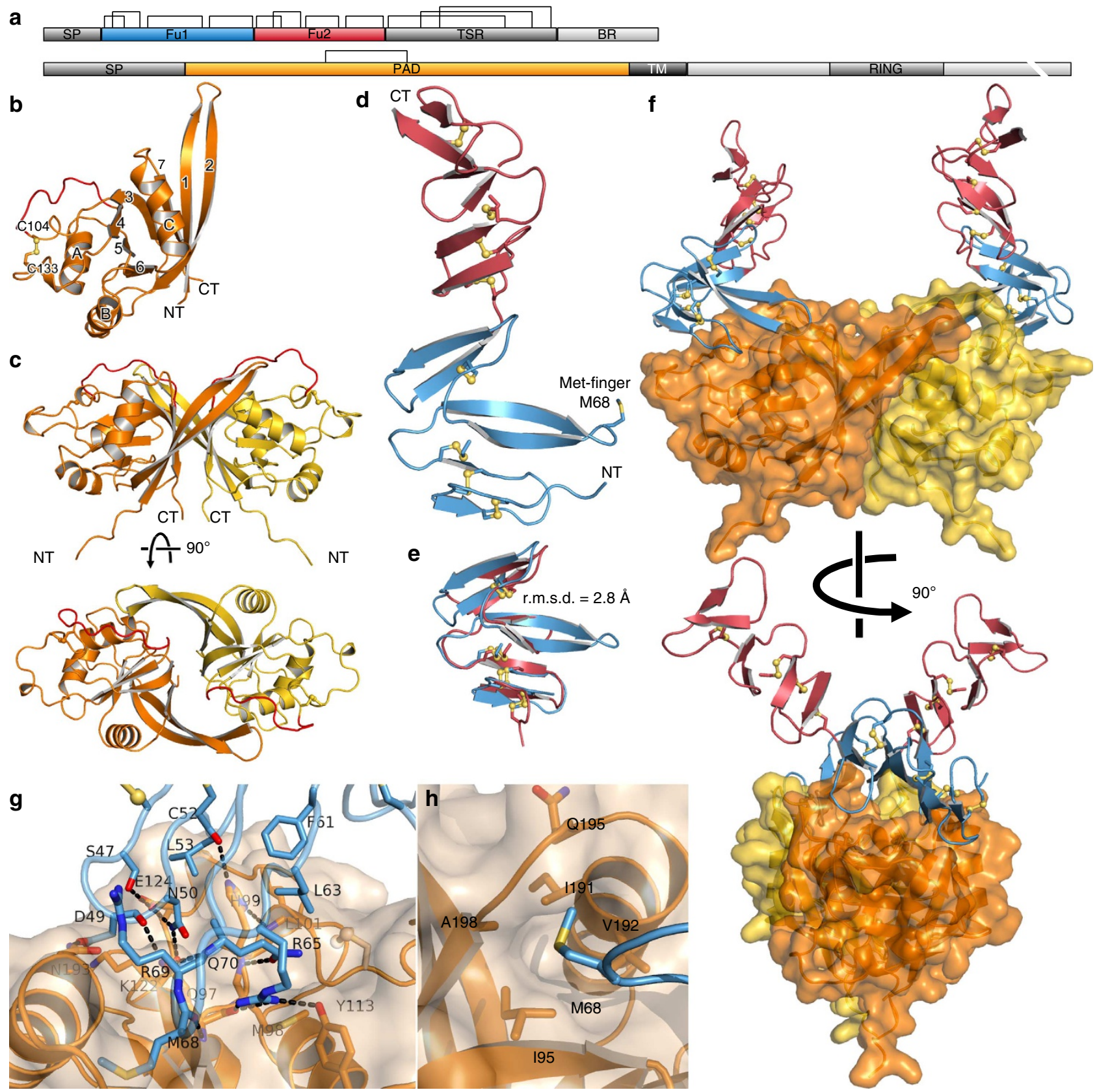

Figure 1 | Unliganded and complexed structures of ZNRF3 and Rspo proteins. (a) Schematic domain organization of Rspo (top) and ZNRF3/RNF43 proteins (bottom) roughly at scale. The domains included in the crystallization constructs are coloured in blue, red and orange. Disulphides are derived from the crystal structure, except for those of the TSR domain of Rspo, which are based on a model ${ }^{48}$. (b) Cartoon representation of the fold of the ZNRF3 ectodomain protomer. $\beta$-strands are numbered and $\alpha$-helices are labelled in alphabetical order from the $N$ to $C$ terminus. (c) Structure of the recurring ZNRF3 $_{\text {ecto }}$ dimer with view parallel to the putative membrane layer and from top towards the membrane. An acidic region with sequence ${ }^{105}$ NNNDEEDLYEY115 is highlighted in red in $\mathbf{b}$ and $\mathbf{c}$. (d) The $x$ Rspo2 $2_{\text {Fu1-Fu2 }}$ structure. Both $\beta$-hairpins and disulphide bridges line up to form a ladder-like structure. The second $\beta$-hairpin of Fu1 contains an exposed methionine side chain. (e) Fu1 and Fu2 share the same architecture, except that the second $\beta$-hairpin of Fu1 is considerably longer. (f) The ZNRF3 ecto $-R_{\text {spo }} 2_{\mathrm{Fu} 1-\mathrm{Fu} 2}$ complex as the same 2:2 symmetric complex in all seven crystallographic observations. Shown are two views parallel to the putative membrane orientation. The RNF43 ${ }_{\text {ecto }}-$ Rspo2 $2_{\text {Fu1-Fu2 }}$ complex resembles one half of this complex

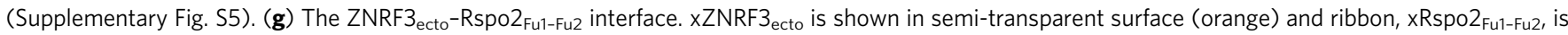
depicted in blue. Residue side chains involved in the interface are shown as sticks and labelled (atom colouring: dark blue, nitrogen; red, oxygen; yellow, sulphur). Dotted lines represent hydrogen bonds. A corresponding stereo figure with final electron density can be found in Supplementary Fig. S6. (h) The Met-finger pocket. Structural features are represented as in $\mathbf{g}$. BR, basic region; PAD, protease-associated domain; SP, signal peptide; TM, transmembrane; TSR, thrombospondin-related domain.

of the two domains, Fu1, is distinguished by the extension of the second $\beta$-hairpin (Fig. 1d,e). This prominent loop presents a solvent exposed methionine (M68) at its tip, which we term the 'Met-finger'.
Structure of liganded complexes of $\mathrm{ZNRF}_{\text {ecto }}$ and RNF43 The crystal structures of the $\mathrm{ZNRF} 3_{\text {ecto }}-\mathrm{Rspo} 2_{\mathrm{Fu} 1-\mathrm{Fu} 2}$ and RNF43 $3_{\text {ecto }}-$ Rspo $2_{\text {Fu1-Fu2 }}$ complexes (Table 1 and Supplementary Table S1) revealed a 1:1 interaction between Fu1 of the Rspo $2_{F u 1-}$ 


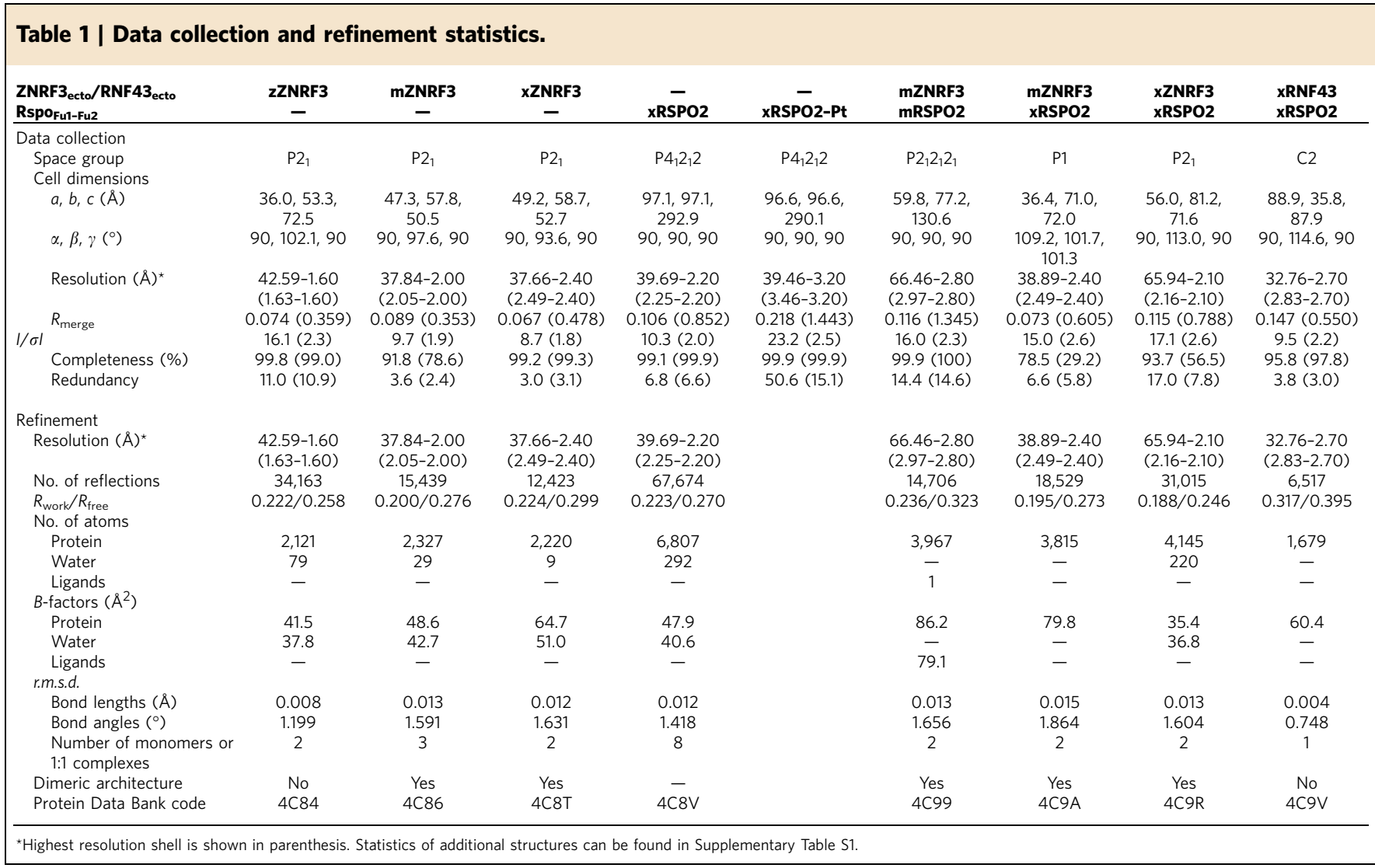

Fu2 and a single ZNRF3 ecto $_{\text {on }}$ RNF43 $3_{\text {ecto }}$ chain (Fig. 1f). As will be discussed below, all complex structures, except for the RNF43 complex, reveal a conserved 2:2 stoichiometry (Supplementary Fig. S5a). The interaction interface between Rspo2 $2_{\mathrm{Fu} 1-\mathrm{Fu} 2}$ and its two receptors RNF43 and ZNRF3 is essentially the same (Supplementary Fig. S5b). It involves an interface area of $990 \pm 105 \AA^{2}$ (Fig. 1g,h, Fig. 3, Supplementary Fig. S6 and Supplementary Table S2). Because of the availability of higher resolution data and multiplicity of data sets, we will first focus on the Rspo2-ZNRF3 interaction. Neither the Rspo2 $2_{\text {Fu1-Fu2 }}$ nor the $\mathrm{ZNRF}_{\text {ecto }}$ dimer show major conformational changes on complex formation (Supplementary Fig. S5). The first, extensive, area of interaction involves hydrophobic interactions interspersed with hydrophilic (complementarily charged) patches contributed by the first two $\beta$-hairpins of the Rspo Ful and the region immediately carboxy-terminal to the $\beta 3$ strand of $\mathrm{ZNRF}_{\text {ecto }}$ (Fig. 1g). The Met-finger at the tip of the second $\beta$-hairpin of Ful nestles into a pocket formed between the $\beta 3$ strand and the $\alpha \mathrm{C}-\beta 7$ loop of the ZNRF $3_{\text {ecto }}$, which is lined with hydrophobic residues (I95, I191, V192, A198; Fig. 1h). The $\alpha \mathrm{C}-\beta 7$ loop is a flexible region in the unliganded $\mathrm{ZNRF}_{\text {ecto }}$ crystal structures and moulds to interface the Rspo Fu1-Fu2 $_{\text {M68 }}$ in the complex. Overall, the ZNRF3 $3_{\text {ecto }}$ dimer structure appears less flexible in the complex structures compared with the unliganded structures. The acidic region of the $\beta 3-\beta 4$ loop (immediately adjacent to $\mathrm{C} 104$ of the disulphide bridge) becomes more ordered in the ligand-bound $\mathrm{ZNRF}_{\text {ecto }}$ structures (Supplementary Fig. S2), probably as a result of electrostatic interactions with a positively charged patch on Rspo Ful (Fig. 3a).

Biophysical and cellular analyses support the structure data. Analytical ultracentrifugation results are consistent with
ZNRF3ecto dimer formation and analyses of several ZNRF3Rspo2 and RNF43-Rspo2 interface mutants (Figs 1g,h and 3) using surface plasmon resonance (SPR)-binding assays confirm the crystallographically determined complex structures (Fig. 4). The single-domain protein $\mathrm{mRspo} 2_{\mathrm{Fu}}$ still bound mZNRF3 $3_{\text {ecto }}$ with high affinity, whereas no detectable binding was measured for $\mathrm{mRspo} 2_{\mathrm{Fu} 2}$ (Fig. $4 \mathrm{~b}$ ). Consistent with the high level of surface residue conservation at the interface (Fig. 3a), the Fu1-Fu2 repeats for all four members of the Rspo family showed binding to $\mathrm{ZNRF}_{\text {ecto }}$ in SPR assays (Fig. 4b). However, the fine-grained differences in the interface-forming residues did impact on the binding affinities; the stronger binding of Rspo2 $2_{\mathrm{Fu} 1-\mathrm{Fu} 2}$ versus Rspo1 $1_{\mathrm{Fu} 1-\mathrm{Fu} 2}$ and $\mathrm{Rspo} 4_{\mathrm{Fu} 1-\mathrm{Fu} 2}$ appeared to be conferred, in part, by the substitution of isoleucine for methionine at the tip of the second $\beta$-hairpin (Fig. 4c). Previously reported genetic and cancer-associated mutations further corroborate the functional significance of the $\mathrm{ZNRF} 3_{\text {ecto }}-\mathrm{Rspo} 2_{\mathrm{Fu} 1-\mathrm{Fu} 2}$ interface as the generic interaction mode for Rspol-4 and ZNRF3/RNF43 (Fig. 4d-g). For example, in Rspo4, the equivalent of the R65W, Q70R and G72R mutations have been reported in inherited anonychia ${ }^{8-10}$. From an analysis of the interaction interface, it is obvious that these mutations are not compatible with ZNRF3/RNF43 binding (Fig. 1g). Functional assays that measure Rspo signalling activity in cells further support the significance of the Rspo-ZNRF3 interface (Fig. 5 and Supplementary Fig. S7). For example, a Met-finger mutation, M68E, which profoundly compromised Rspo-ZNRF3 interaction in SPR assays, exhibited much weaker signalling activity, whereas a conserved substitution, M68I, showed slightly reduced binding to ZNRF3 and relatively normal (or slightly reduced) signalling capacity (Fig. 5d). Other interface mutants in Fu1, including the anonychia-associated mutations R65W, Q70R and G72R, as well as N50R, each exhibited weakened signalling 

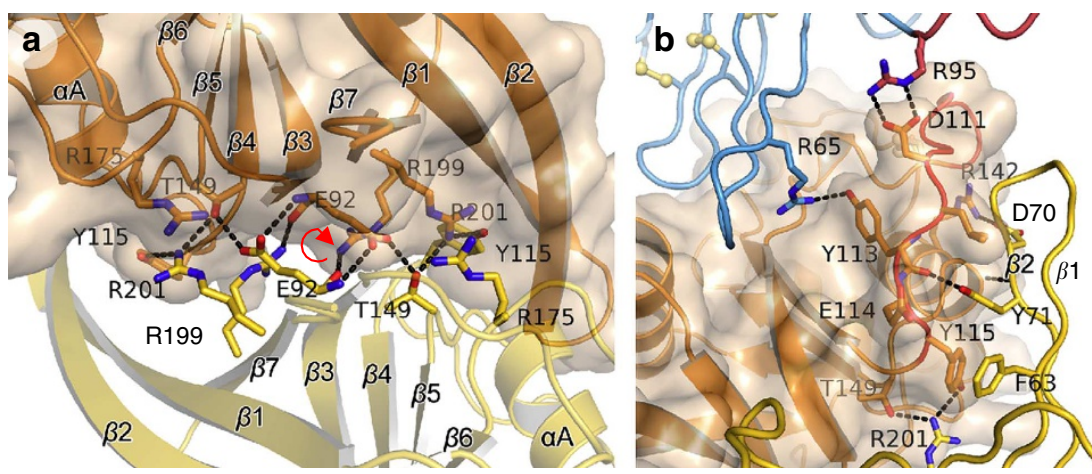

Figure 2 | Dimerization interface of $\mathbf{x Z N R F}_{\text {ecto }}$ (a) View along the twofold axis away from the putative membrane. (b) $\mathrm{xZNRF}_{\mathrm{ecto}}-\mathrm{xRsp \textrm {F } _ { \mathrm { Fu } } - \mathrm { Fu } 2} \mathrm{Complex}$ with close-up view onto the $\beta 1-\beta 2$ hairpin arm ('clamp') embracing the respective other protomer. This interface is stabilized by binding of Rspo to ZNRF3 and subsequent structuring of the acidic region of the $\beta 3-\beta 4$ loop drawn in red. Residue numbers refer to mouse proteins.

a

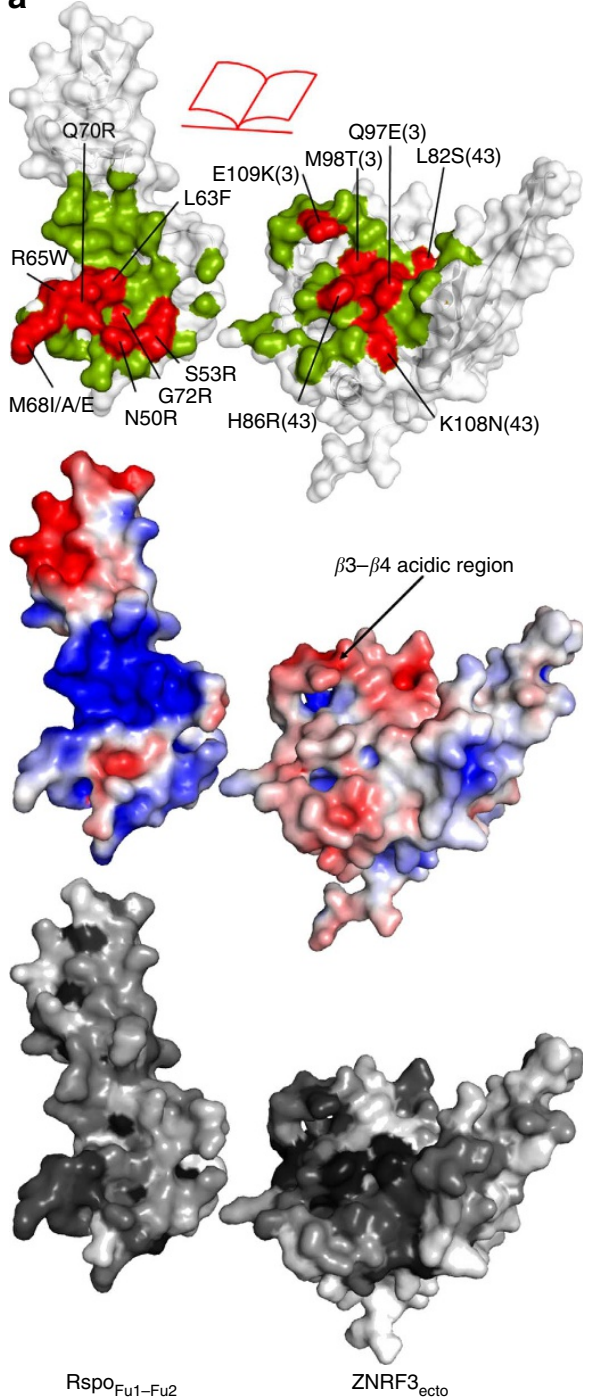

b
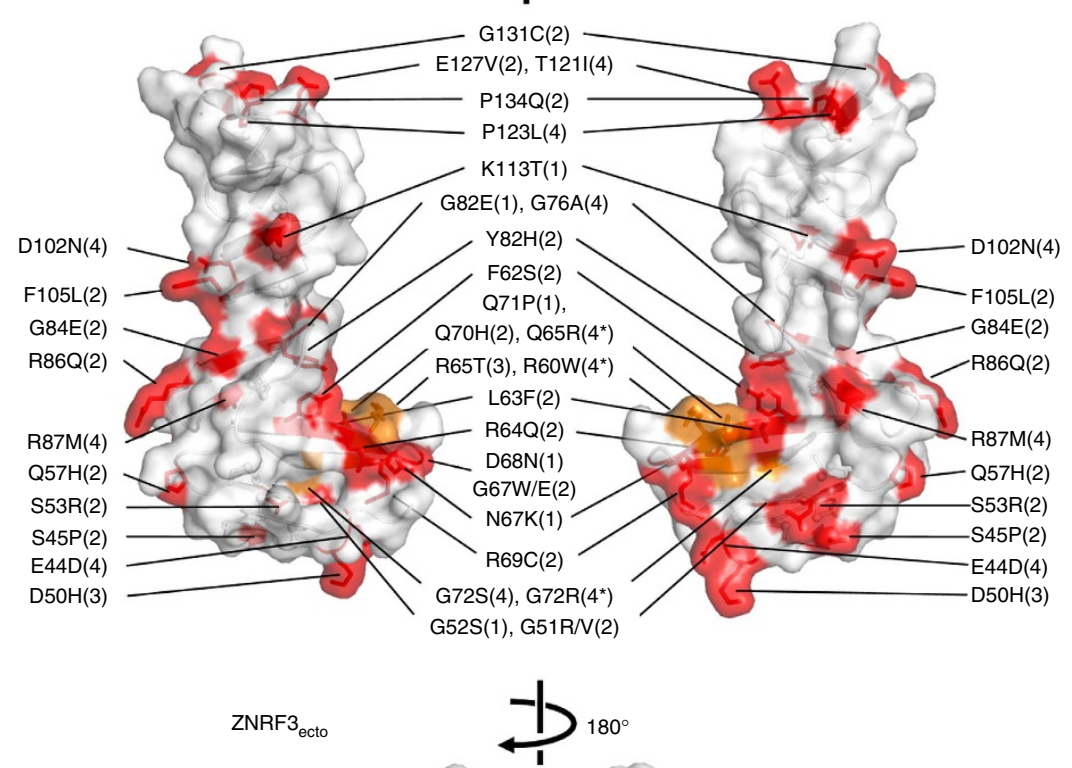

Figure 3 | Characteristics of the ZNRF3 dimer and Rspo-ZNRF3 complex interfaces. (a) An open book view of the ZNRF3-Rspo interface. The surface contributing to the interface is coloured green on $\mathrm{ZNRF}_{\text {ecto }}$ and Rspo Fu1-Fuz; within this, surface mutants tested in this study are highlighted in red (top). Rspo and ZNRF3 ${ }_{\text {ecto }}$ coloured by electrostatic surface potential from red (acidic) to blue (basic) (middle). Sequence conservation across species coloured from white (not conserved) to black (conserved). (b) Disease-related mutations are plotted onto the molecular surface of Rspo (top) and ZNRF3/ RNF43 (bottom), and are concentrated at the Rspo-ZNRF3/RNF43 interaction interface. Tumour-associated missense mutations derived from the cosmic database (http://cancer.sanger.ac.uk/cancergenome/projects/cosmic/) are shown in red and missense mutations causal for congenital anonychia on RSPO4 are shown in orange. Sites in orange on ZNRF3 are mutations of RNF43 that map to the dimer interface of ZNRF3. Numbers 1-4 in parentheses indicate mutations found in RSPO1 to RSPO4 (top). Number 3 and 43 in parentheses indicate mutations found in ZNRF3 and RNF43, respectively (bottom). 
ability that correlated with reduction in binding to ZNRF3 (Fig. 5e).

Dimerization propensity of ZNRF3 versus monomeric RNF43. For all $\mathrm{ZNRF} 3_{\text {ecto }}-$ Rspo $2_{\mathrm{Fu} 1-\mathrm{Fu} 2}$ complex structures we determined (from five different combinations of species and crystal forms), the dimer found in most of the unliganded $\mathrm{ZNRF}_{\text {ecto }}$ crystal structures reoccurs (Fig. 1f, Supplementary Fig. S5 and Supplementary Table S2). The overall assembly thus comprises a 2:2 complex of $\mathrm{ZNRF}_{\text {ecto }}-\mathrm{Rspo} 2_{\mathrm{Fu} 1-\mathrm{Fu} 2}$. The 2:2 complex resembles a crab with the $\mathrm{ZNRF} 3_{\text {ecto }}$ dimer forming the body from which the two Rspo $2_{\mathrm{Fu} 1-\mathrm{Fu} 2}$ ligands diverge, without

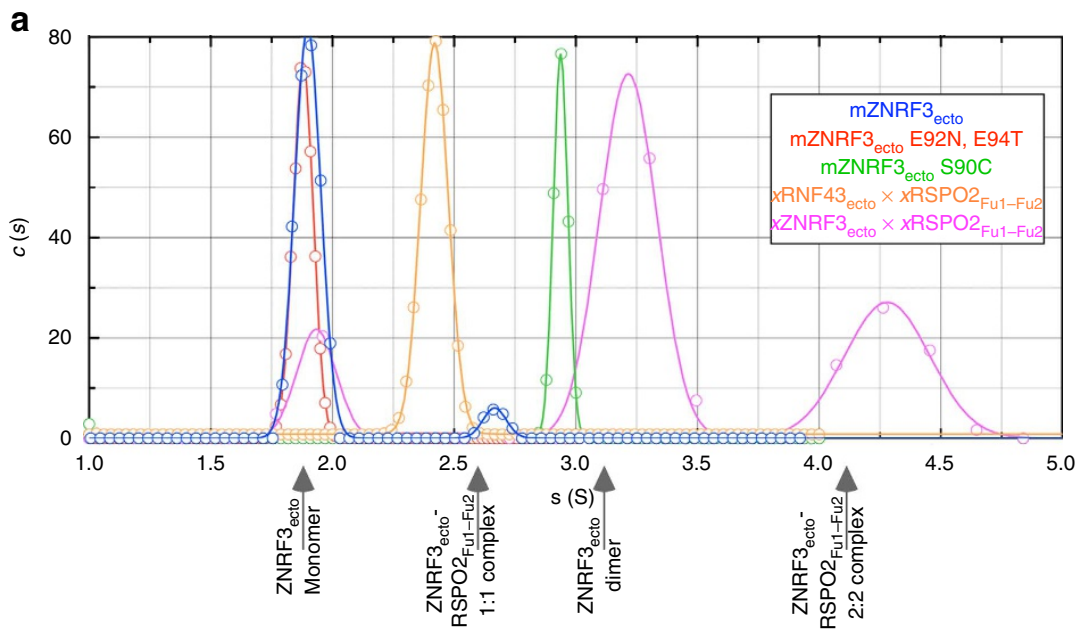

b

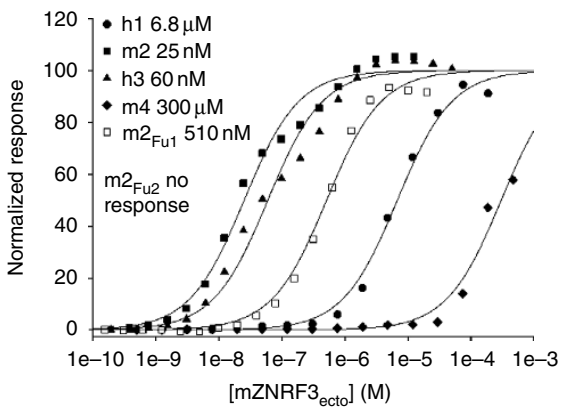

C

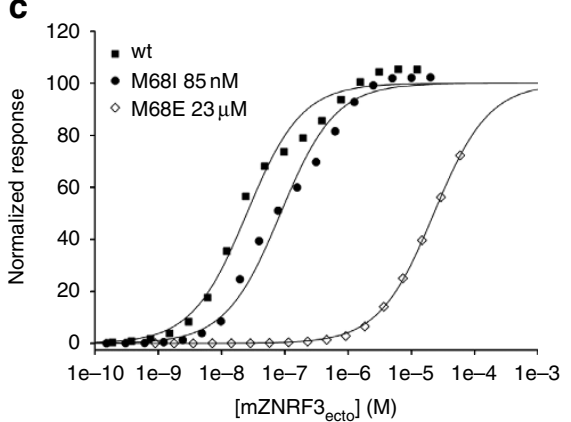

f

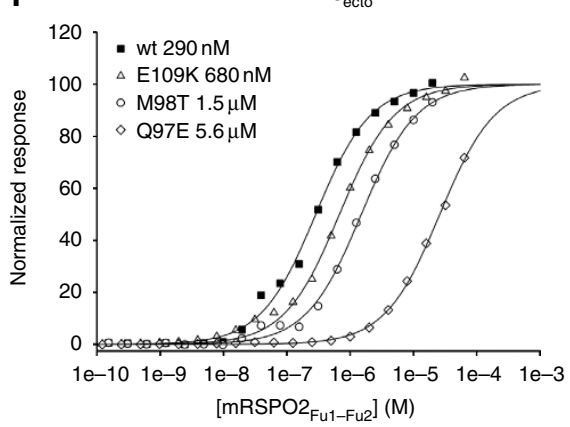

i

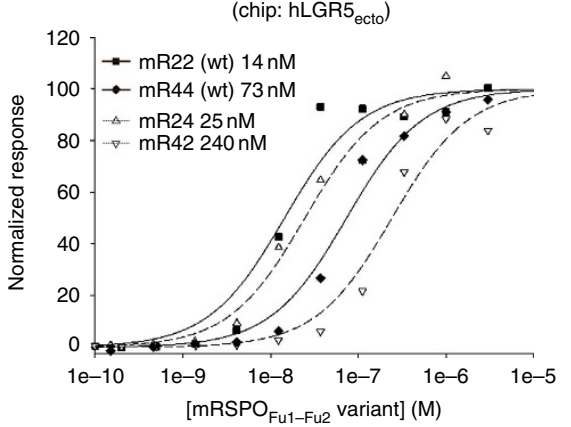

d

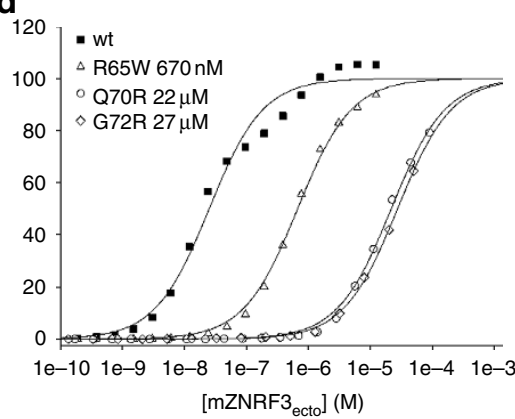

g hRNF43 ${ }_{\text {ecto }}$

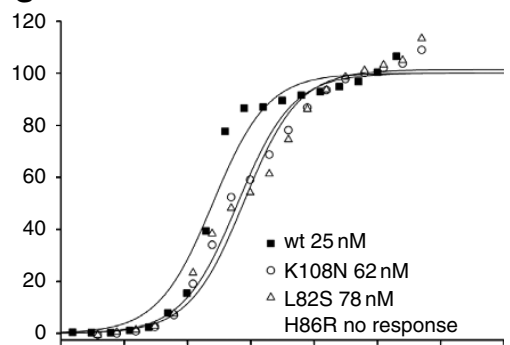

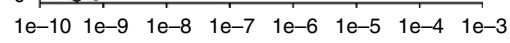
$\left[\mathrm{MRSPO} 2_{\mathrm{Fu} 1-\mathrm{Fu} 2}\right.$ (M)
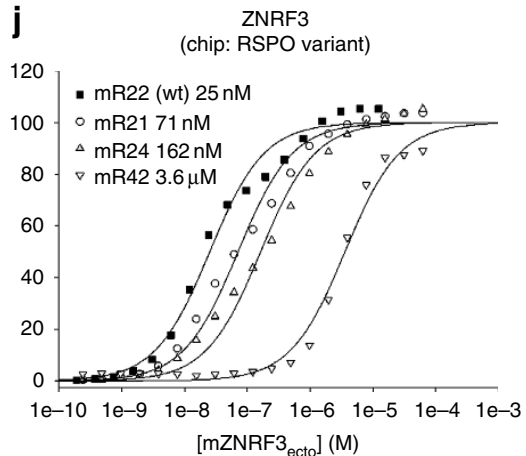

e

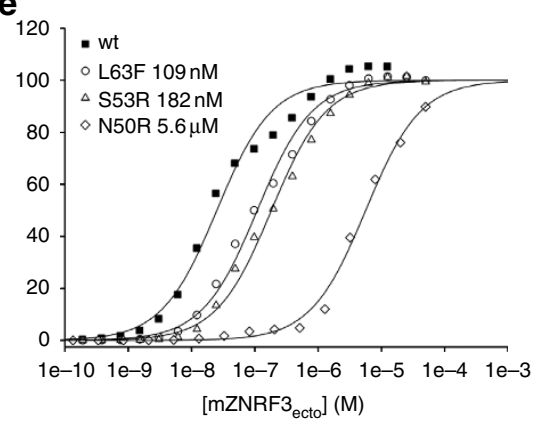

h mZNRF $_{\text {ecto }}$

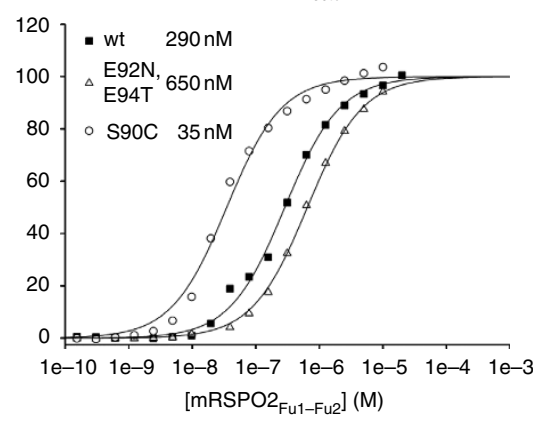

K mZNRF $_{\text {ecto }}$

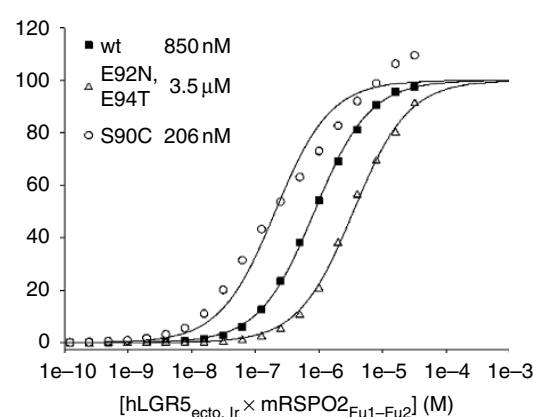


interacting with each other, as the pincers. In contrast, our single structure of RNF43 in complex with Rspo2 $2_{\mathrm{Fu} 1-\mathrm{Fu} 2}$ displays no dimeric architecture.

Dimerization of ZNRF3 $3_{\text {ecto }}$ is weak in solution and the protein did not behave as a dimer in gel filtration. Still, a propensity of $\mathrm{ZNRF}_{\text {ecto }}$ to dimerize was evident from analytical ultracentrifugation data. Broad peaks of $\mathrm{ZNRF}_{\text {ecto }}$ from ultraviolet absorbance data were highly indicative of a rapid equilibrium of self-association. In sedimentation velocity plots using the faster interference optics traces of a dimer could be detected (Fig. 4a). This rapid dimerization was not observed when a glycosylation site E92N, E94T was engineered into the dimerization interface observed in the crystal structures. Formation of the observed crystallographic ZNRF3 dimer in solution is further supported by the observation of almost quantitative spontaneous crosslinking of the S90C variant of $\mathrm{mZNRF}_{\text {ecto }}$ that introduces a cysteine close to the dimer symmetry axis (Supplementary Fig. S8). A crystal structure of this variant at $2.1 \AA$ shows that this mutation and crosslinking is easily accommodated, requiring only minor backbone distortions (Supplementary Table S1 and Supplementary Fig. S8d).

Formation of the ZNRF3 $3_{\text {ecto }}-$ Rspo2 $2_{\text {Fu1-Fu2 }}$ complex also leads to increased dimerization in solution (Fig. 4a). An explanation for this is found by careful analysis of the crystal structures. As outlined before, ligand binding leads to a structuring of the acidic region of the $\beta 3-\beta 4$ loop (red in Figs $1 b, c$ and $2 b$ ). This same region of the $\beta 3-\beta 4$ loop also interacts with the $\beta 1-\beta 2$ hairpin in the opposing subunit of the $\mathrm{ZNRF}_{\text {ecto }}$ dimer (Fig. 2b). Notably, the $\beta 1-\beta 2$ hairpin shows less conformational variation in the liganded ZNRF3 ${ }_{\text {ecto }}$ dimer structures, always maintaining a tight embrace (Supplementary Figs S2b and S5a), consistent with the $\mathrm{Rspo}_{\mathrm{Fu} 1-\mathrm{Fu} 2}$ interactions contributing an indirect stabilizing effect on the dimer via the $\beta 3-\beta 4$ loop. This stabilizing effect provides an explanation for our results showing that Rspo2 $2_{\mathrm{Fu1}-\mathrm{Fu} 2}$ bound weaker to monomerized $\mathrm{mZNRF}_{\text {ecto }}$ E92N, E94T but stronger to predimerized $\mathrm{mZNRF}_{\text {ecto }} \mathrm{S} 90 \mathrm{C}$ than to the wt mZNRF3 ${ }_{\text {ecto }}$ (Fig. 4h).

No dimer is observed in solution for RNF43 $3_{\text {ectoo }}$ even after binding to $\mathrm{mRspo} 2_{\mathrm{Fu} 1-\mathrm{Fu} 2}$ (Fig. 4a), and we also see only a minor propensity for spontaneous cysteine crosslinking of the P77C (corresponding to $\mathrm{S} 90 \mathrm{C}$ of $\mathrm{mZNRF} 3_{\text {ecto }}$ ) variant of hRNF43 ecto (Supplementary Fig. S8b). We note that the residues involved in the dimerization interface of ZNRF3, albeit conserved within the ZNRF3 family, are not conserved between ZNRF3 and RNF43 (Supplementary Fig. S5c). Furthermore, two glycosylation sites exist in RNF43 and map to the acidic region of the $\beta 3-\beta 4$ loop and the $\beta 1-\beta 2$ clamp (Supplementary Figs S2 and S5). These sites are not resolved in the RNF43 complex but might sterically hamper dimerization.

Rspo interacts with LGRs via Fu2. Fu2, similar to Fu1, is essential for Rspo signalling function ${ }^{1}$ (Supplementary Fig. S1).
We therefore suspected that Fu2 might be involved in binding to other components of the Rspo receptor complex, such as LGR4/5/6. Indeed, although Rspo1, Rspo1 $1_{\mathrm{Fu} 1-\mathrm{Fu} 2}$ and Rspo1 ${ }_{\Delta \mathrm{Fu} 2}$ were each co-immunoprecipitated with RNF43, confirming that Fu1 is critical for binding to ZNRF3/RNF43, Rspo1, Rspo1 ${ }_{\mathrm{Fu1}-\mathrm{Fu} 2}$ and $\mathrm{Rspo}_{\triangle \mathrm{Fu} 1}$ each immunoprecipitated LGR4, indicating that Fu2 is the primary binding site for LGR receptors (Fig. 6a,b). Consistent with these co-immunoprecipitation data, Rspo2 $2_{\mathrm{Fu} 1-\mathrm{Fu} 2}$ simultaneously bound to ZNRF3 and LGR5 in SPR binding assays (Fig. 6c-e) with the monomerized $\mathrm{ZNRF}_{\text {ecto }}$ binding weaker and the dimerized $\mathrm{S} 90 \mathrm{C}$ variant binding stronger to a preformed LGR $5_{\text {ecto }}-$ Rspo $\mathrm{Fu}_{1-\mathrm{Fu} 2}$ complex (Fig. 4k). Our data therefore support the model of a ZNRF3/RNF43-Rspo-LGR4/5/6 complex assembled through ZNRF3/RNF43-Rspo Fu1 and Rspo $_{\mathrm{Fu} 2}-\mathrm{LGR} 4 / 5 / 6$ interactions (Fig. 7).

Rspo-ZNRF3/RNF43 interaction determines signalling potency. Although there is a clear requirement for both Furin domains in Rspo ternary complex formation and functional activation of the Wnt pathway, our results point to the ability of $\mathrm{Rspo}_{\mathrm{Fu} 1}$ to recruit ZNRF3/RNF43 as the major determinant of activity (for example, Figs $4 \mathrm{~b}$ and $5 \mathrm{~b}$ ). In biophysical assays, wildtype mRspo2 and -4 proteins, as well as their Fu1/Fu2 chimeras, bound with nanomolar affinity to hLGR5 $5_{\text {ecto }}$ (Fig. 4 i), further suggesting that engagement of LGR is not the efficiency-determining step. Cellular assays using Rspo2 Fu1/Fu2 chimeras showed that a Fu1 repeat from a 'strong' Rspo (that is, Rspo2 or -3) was sufficient to induce a higher Wnt response (Fig. 5c). On the other hand, in spite of an 80 -fold $(300 \mu \mathrm{M}>3.6 \mu \mathrm{M})$ increase in binding efficiency to $\mathrm{ZNRF}_{\text {ecto }}$, replacement of Fu2 of Rspo4 by that of a 'strong' Rspo was not able to enhance Wnt signalling when expressed at comparable levels (Fig. 5c). Hence, it can be concluded that functional efficiency of the four Rspo ligands is largely based on their ability to recruit ZNRF3 or RNF43 via Ful into a complex with LGRs.

\section{Discussion}

In combination, the structural, biophysical and cell-based studies we report here for the ZNRF3/RNF43-Rspo system reveal two modes of interaction: receptor-ligand and receptor dimer. For the ligand-receptor mode, our data define a generic architecture for the interaction between the Rspo ligands, and the ZNRF3 and RNF43 transmembrane E3 ubiquitin ligases that is conserved across evolution from fish to human. Indeed, the differences in binding affinities, from highest affinity for Rspo2 to lowest for Rspo4, appear to mirror the trend in biological activity of the four Rspo proteins (reviewed in ref. 3). Our results highlight the role of the Ful domain of the Rspo protein in ZNRF3/RNF43 binding. Both $\mathrm{Fu}$ domains together have been implicated in Rspo signalling. The primary role of Fu1 in ZNRF3/RNF43 binding leaves a substantial surface available for the Rspo to mediate

Figure 4 | Biophysical characterization of the ZNRF3ecto dimer and interface mutants. (a) Sedimentation velocity experiments. A plot of $c(s)$

(in arbitrary units) against $\mathrm{s}$ (in svedbergs). Shown in each case are individual data points and the fit of an appropriate number of Gaussian distributions. All samples were adjusted to a concentration of $350 \mu \mathrm{M}$. Also shown arrowed are the expected sedimentation coefficients for the different complexes observed in the crystal structures as predicted using HYDROPRO (see Methods). (b-h) SPR experiments using mZNRF3 ${ }_{\text {ecto }}$ (b-e) or mRspo2 $2_{\text {Fu1-Fu2 }}$ (f-h)

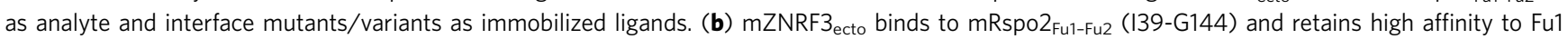
(I39-R95) but not to Fu2 (A94-G144). Fu1-Fu2 polypeptides of human or mouse homologues (hRspo1: I32-S143, hRspo3: R32-H147, mRspo4: T29-Q136) bind with different affinity to mZNRF3 ${ }_{\text {ecto }}$ (c) Mutations of the Met-finger impact affinity. (d) Anonychia mutations of RSPO4 introduced to mRspo2 $2_{\text {Fu1-Fu2 }}$ drastically impair binding. (e) Three additional interface mutants of which two (L63F and S53R) have been found in tumour tissues. (f) As the immobilized ligand mZNRF3 binds with lower affinity to the $\mathrm{mRspo}_{2 \mathrm{Fu} 1-\mathrm{Fu} 2}$ analyte. Of the three interface mutants, two (E109K and M98T) have been identified in tumour tissues. (g) Three interface mutants of hRNF3 ${ }_{\text {ecto }}$ have been identified in tumours, one of which completely disrupts binding. (h) Binding of

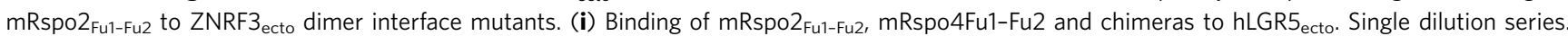

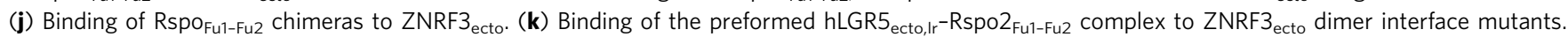



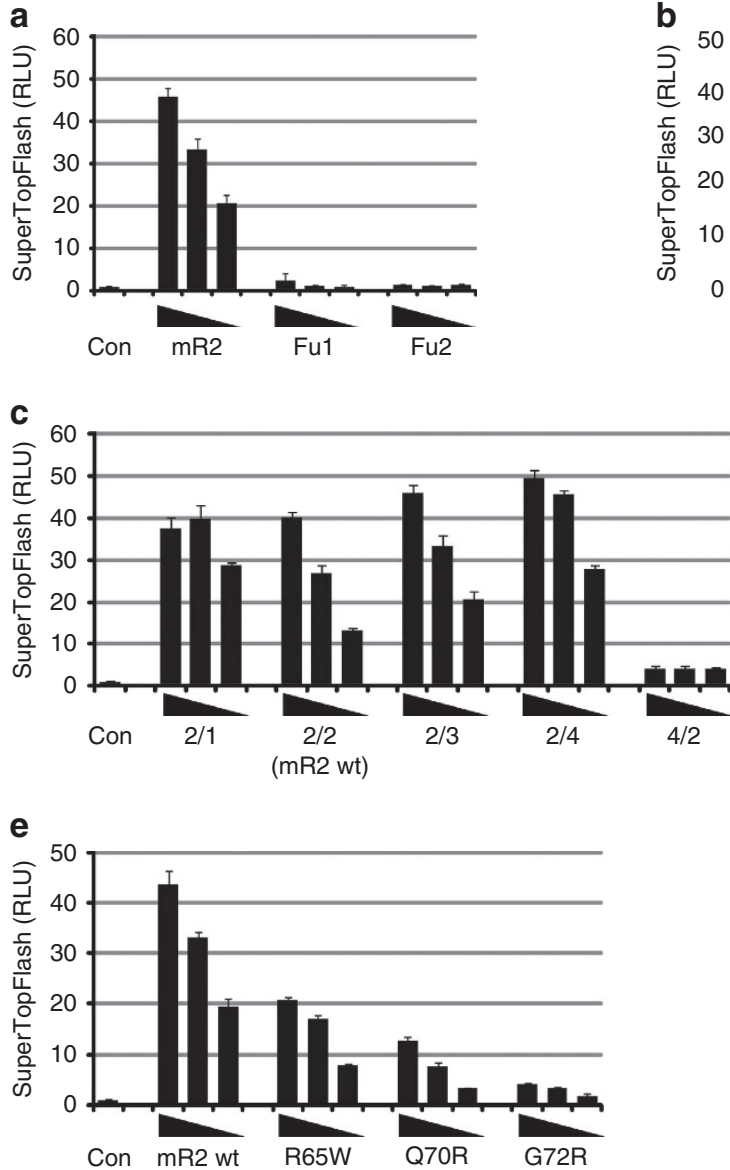

g
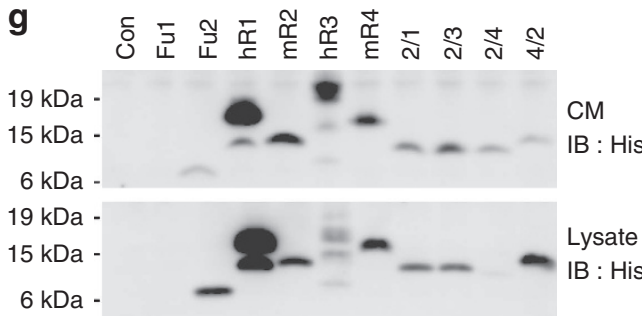
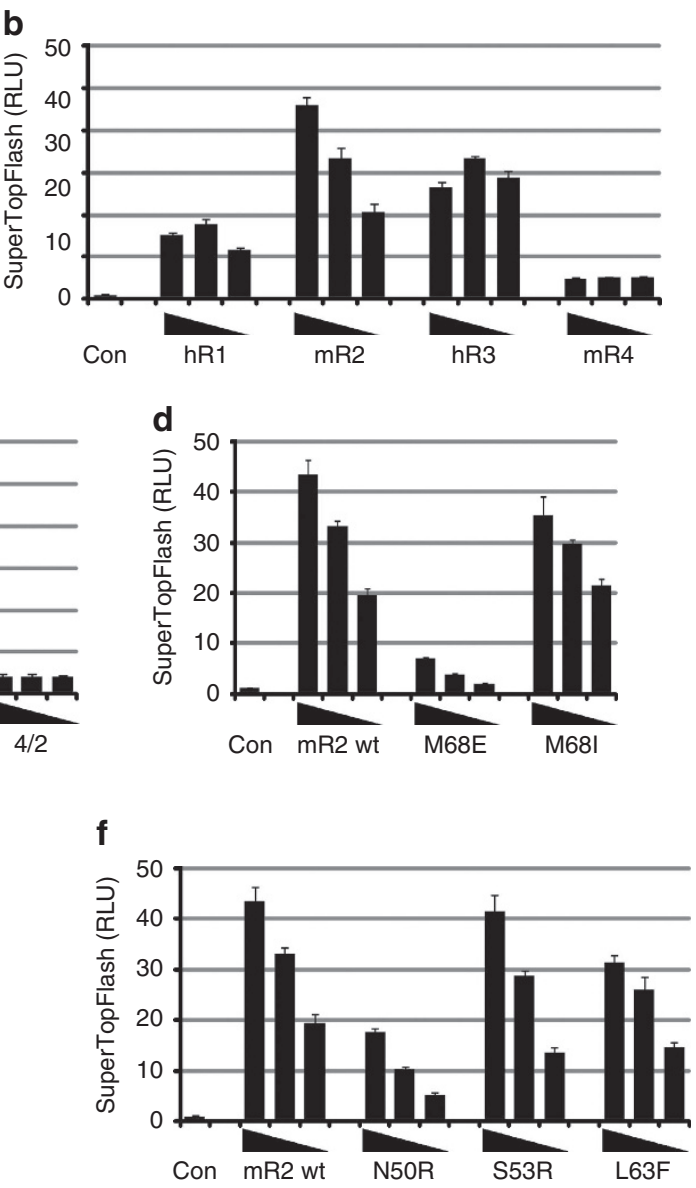

h
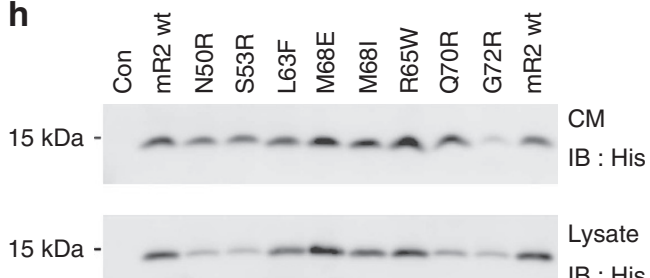

IB : His

Figure 5 | Activation of the Wnt pathway assayed by the SuperTopFlash reporter. (a-f) Co-transfected decreasing doses (25, 5 and $1 \mathrm{ng}$ ) of His-tagged R-spondin constructs used for SPR experiments in Fig. 4. Error bars represent s.d. from three replicates. (g,h) Western blots showing expression levels of the His-tagged R-spondin constructs from whole-cell lysate and conditioned media (CM). Expression for mRspo2 Fu1-His was poor and below the level of detection; however, the individual Fu1 domain from RSPO1 was detected by western blotting and produced identical results (Supplementary Fig. S1b,c). RLU, relative luciferase units.

formation of a three component complex involving ZNRF3/ RNF43, Rspo and LGR4/5/6 as postulated ${ }^{16}$. Indeed, our coimmunoprecipitation results focus LGR4/5/6-binding activity onto $\mathrm{Fu} 2$, consistent with Rspo proteins acting as complex assemblers (Fig. 7). Whilst we were preparing our paper for publication, several crystal structures of Rspo1 $1_{\mathrm{Fu} 1-\mathrm{Fu} 2}$ in complex with LGR4/5 $5_{\text {ecto }}$ and a single Rspo1 $1_{\text {Fu1-Fu2 }}-$ LGR5 $5_{\text {ecto }}-$ RNF43 $3_{\text {ecto }}$ complex were reported ${ }^{25-28}$, which fully support this notion.

Unexpectedly, our analyses reveal a dimerization mode for ZNRF3 $3_{\text {ecto }}$. The conservation of ZNRF3 ectodomain dimerization across evolution from fish to mammals suggests that this interaction has some role in the mechanism of action of ZNRF3. It is also noteworthy that three cancer-associated mutations reported for RNF43 map to the corresponding dimer interface observed in the ZNRF3 $3_{\text {ecto }}$ crystal structures (Fig. 3b), suggesting the characteristics of this surface have functional relevance in
RNF43 as well. Many members of the E3 RING ubiquitin ligase superfamily have been reported to require dimerization for function (reviewed in ref. 29), a conclusion supported by recent insights into the mechanism of action of the RING ligase RNF4 (ref. 30). In ZNRF3, the ectodomain may, alongside cytoplasmic regions, contribute to functionally essential RING domain dimerization. However, neither our biophysical measurements nor our structural data for an RNF43 ectodomain in complex with Rspo 2 provide any evidence of a similar dimerization mode for RNF43; a finding that argues against ectodomain dimerization having a central role in ligase activity. The newly reported structure of the 1:1:1 complex of Rspo1-LGR5-RNF43 also reveals no RNF43 dimer ${ }^{25}$. Intriguingly at the level of a simple modelling exercise, the ternary complex architecture appears compatible with ZNRF3 dimerization (Fig. 7). All of the currently available structures are compatible with a dimeric ZNRF3 as 
a

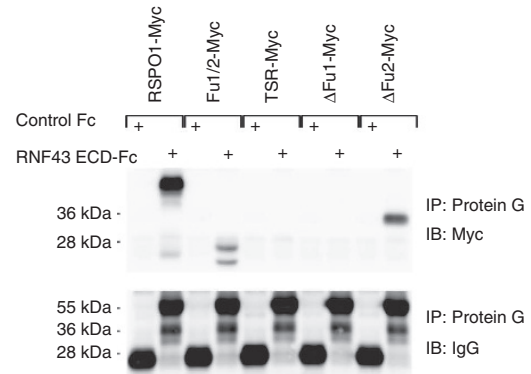

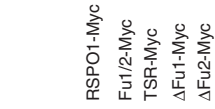

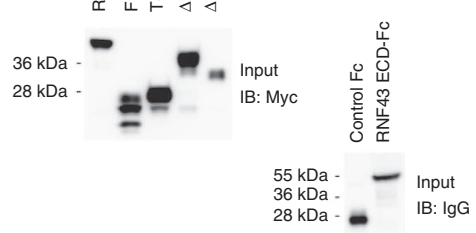

b

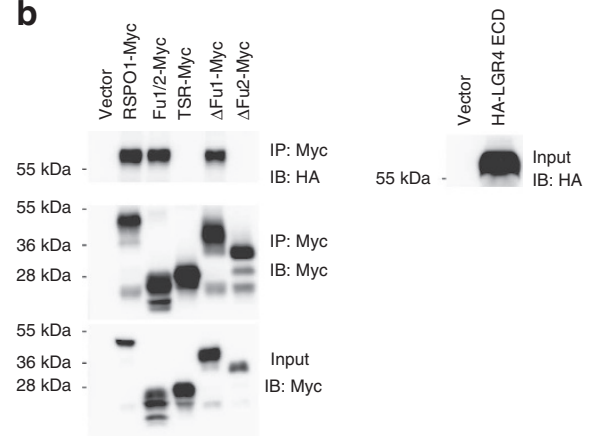

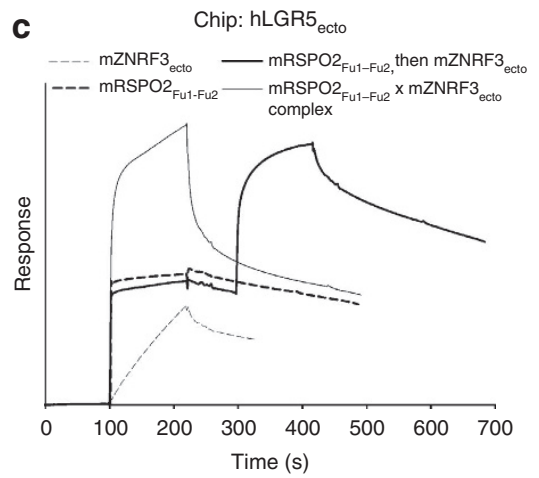
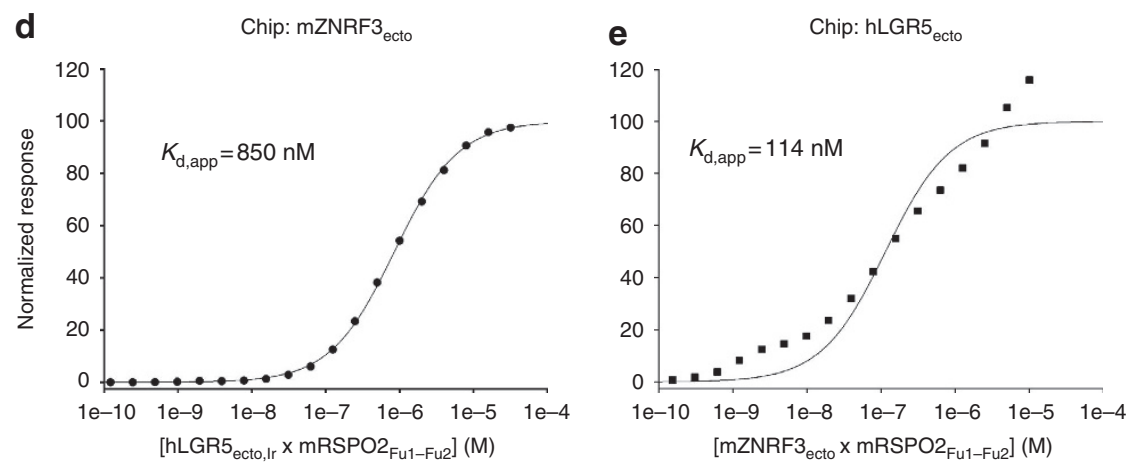

Figure 6 | The LGR-Rspo-ZNRF3/RNF43 ternary complex. (a) RNF43 interacts with Fu1 of human Rspo1. The secreted RNF43 ectodomain coimmunoprecipitated Rspo1 and its derivatives, Rspo1 Fu1-Fu2 $_{2}$ and Rspo1 ${ }_{\triangle \mathrm{Fu} 2}$, but neither RSPO1 ${ }_{\triangle \mathrm{Fu} 1}$ nor Rspo1 TSR $_{\text {in }}$ inditioned media (CM; left). RNF43 is IgG-tagged, whereas Rspo1 and derivatives are Myc-tagged, and their secretion levels in CM were also examined (right). (b) LGR4 interacts with Fu2 of

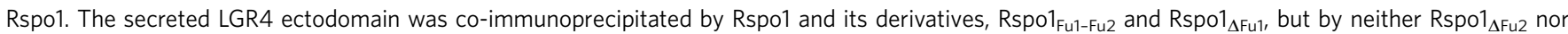
Rspo1 $_{\text {TSR }}$ in CM (left). Secreted LGR4 is HA-tagged and its secretion in CM was examined as were Rspo1 and derivatives (right). (c) Step-by-step ternary complex assimilation. hLGR5ecto (R32-G557) was immobilized on an SPR chip, followed by injections of $10 \mu \mathrm{M}$ solutions of $\mathrm{mZNRF3} 3_{\text {ector }} \mathrm{mRSPO}_{\text {Fu1-Fu2, }}$ mRSPO2 $2_{\text {Fu1-Fu2 }}$ followed by mZNRF3 $3_{\text {ecto }}$ or a preformed mZNRF3 ecto $\times$ mRSPO2 Fu1-Fu2 $_{\text {complex. }}$ ZZNRF3 $3_{\text {ecto }}$ shows some direct interaction with LGR5 characterized by a slow on-rate (thin dashed line). Binding is much faster if LGR5 is first saturated with mRSPO2 Fu1-Fu2 (thick solid line). Similar responses are observed when a 1:1 complex of $\mathrm{mRSPO} 2_{\mathrm{Fu} 1-\mathrm{Fu} 2} \times \mathrm{mZNRF}_{\text {ecto }}$ is injected. (d) Saturation of immobilized mZNRF3 $3_{\text {ecto }}$ with the hLGR5 $5_{\text {ecto }} \times$ mRSPO2 $_{\text {Fu1-Fu2 }}$ complex that was stable in gel filtration. Ir, loop removed: A488-H537 $\rightarrow$ NGNNGD. (e) Saturation of immobilized hLGR5 with the $\mathrm{mZNRF}_{\text {ecto }} \times \mathrm{mRSPO}_{\mathrm{Fu} 1-\mathrm{Fu} 2}$ complex that was stable in gel filtration. Single dilution series.

reported here. However, we note that none of the reported crystallographic LGR $4 / 5_{\text {ecto }}$ dimers would be compatible with simultaneous binding of Rspo proteins to both LGR4/5/6 and ZNRF3/RNF43. Our observation is suggestive of Rspo functioning to sequester ZNRF3 dimers into a complex with LGR4/5/6. Thus, we may speculate that the difference in oligomeric state of the ZNRF3 and RNF43 ectodomains points to some yet to be ascertained difference in their function or regulation. We note that sequence conservation of the ectodomain within the ZNRF3 subfamily is far greater than that within the RNF43 subfamily (Supplementary Table S5), supporting the notion that for ZNRF3 function or regulation additional features such as ligand-induced dimerization may be important.

ZNFR3 and RNF43, alongside the Rspo proteins, have emerged as a system with significant therapeutic potential for a number of pathological processes. The insights into molecular mechanism presented here open up new avenues to explore for possible manipulation of this system.

\section{Methods}

Large-scale expression of $\mathbf{Z N R F}_{\text {ecto }}$ and $\mathbf{R S P O}_{\text {Fu1-Fu2. Synthetic com- }}$ plementary DNA clones for ectodomains of mouse and zebrafish ZNRF3 and Xenopus RNF43 were obtained from Invitrogen/Geneart (Germany). All other template cDNAs were from the I.M.A.G.E. library.

Xenopus, mouse and zebrafish ZNRF3 ectodomains (residues E25-D191, K53-L205 and K30-R181, respectively), Xenopus RNF43 ectodomain (T28-D192), as well as Xenopus and mouse Rspo2 $2_{\mathrm{Fu} 1-\mathrm{Fu} 2}$ constructs (residues G35-D143, N37 or
I39-G144) were cloned into the pHLsec vector ${ }^{31}$ that encodes for a C-terminal His6-tag (His10-tag for mRSPO2 ${ }_{\mathrm{Fu} 1-\mathrm{Fu} 2}$ ). Proteins were expressed separately or after co-transfection in HEK293T cells seeded into roller bottles. For preparation of seleno methionine (SeMet)-labelled $x R S P O 2_{\mathrm{Fu} 1-\mathrm{Fu} 2}$, the cells were washed $24 \mathrm{~h}$ after transfection with PBS $(2 \times 35 \mathrm{ml}$ per roller bottle $)$ and the medium was changed to methionine-free DMEM complemented with $2 \%$ dialysed fetal bovine serum and $40 \mu \mathrm{g} \mathrm{ml}^{-1}$ SeMet. After 4-8 days expression, the medium was collected and cleared by centrifugation and filtration. The buffer was exchanged to $10 \mathrm{mM}$ Tris/ $\mathrm{HCl}, \mathrm{pH} 8.0,500 \mathrm{mM} \mathrm{NaCl}$ using hollow-fibre ultrafiltration. Proteins were purified using $\mathrm{Ni} 2^{+}$-charged $5 \mathrm{ml}$ HisTrap FF columns from GE. Before sample loading, $25 \mathrm{mM}$ imidazole was added to suppress unspecific binding. The elution buffer contained $1 \mathrm{M}$ imidazole in binding buffer. Individual proteins were subjected to gel filtration on S200 16/60 pg columns (GE Healthcare) equilibrated with $10 \mathrm{mM}$ HEPES/NaOH, pH 7.5, $150 \mathrm{mM} \mathrm{NaCl}$. ZNRF3 ecto $_{\text {-RSPO2 }}$ Fu1-Fu2 complexes were obtained by coexpression or by mixing of equimolar amounts followed by gel filtration. Because of solubility issues of the complexes, the $\mathrm{NaCl}$ concentration of the gel filtration buffer was increased to $250 \mathrm{mM}$.

Crystallization and data collection. Concentrated proteins were subjected to sitting drop vapour diffusion crystallization trials employing a Cartesian Technologies pipetting robot, and usually consisted of $100-300 \mathrm{nl}$ protein solution and $100 \mathrm{nl}$ reservoir solution ${ }^{32}$. Crystal form I of apo xRSPO2 $2_{\mathrm{Fu}-\mathrm{Fu} 2}$ appeared in $100 \mathrm{mM}$ Bis-Tris/HCl, pH 6.3, $200 \mathrm{mM}$ ammonium sulphate (AS) and $1.2 \mathrm{M}$ tartrate, $\mathrm{pH} 7.5$, at a protein concentration of $37.5 \mathrm{mg} \mathrm{ml}^{-1}$. Crystals of form II appeared in $100 \mathrm{mM}$ cacodylate and $1 \mathrm{M}$ sodium citrate, final $\mathrm{pH} \mathrm{7}$, at a protein concentration of $24 \mathrm{mg} \mathrm{ml}^{-1}$. zZNRF3 $3_{\text {ecto }}$ crystallized in $20 \%(\mathrm{w} / \mathrm{v})$ PEG3350, $200 \mathrm{mM} \mathrm{CaCl}_{2}$ (form I) or 25\% (w/v) PEG3350, $100 \mathrm{mM}$ Bis-Tris, pH 5.5 (form II) Crystals of $\mathrm{mZNRF}_{\text {ecto }}$ appeared at sample concentrations of $49 \mathrm{mg} \mathrm{ml}^{-1}$ in $25 \%$ (w/v) PEG3350, $200 \mathrm{mM} \mathrm{MgCl}_{2}$ and $100 \mathrm{mM}$ Tris, $\mathrm{pH} \mathrm{8.5,} \mathrm{(form} \mathrm{I);} 20 \%$ (w/v) PEG3350, 5\% (w/v) low-molecular-weight polyglutamic acid and $100 \mathrm{mM}$ Tris, $\mathrm{pH}$ 7.8, (form II); $20 \%$ (w/v) PEG8000, $200 \mathrm{mM} \mathrm{MgCl}_{2}$ and $100 \mathrm{mM}$ Tris, $\mathrm{pH} 8.5$ (form III); or crystallized alone out of a complex sample with bovine RSPO2 $2_{\mathrm{Fu} 1-\mathrm{Fu} 2}$ at 
a
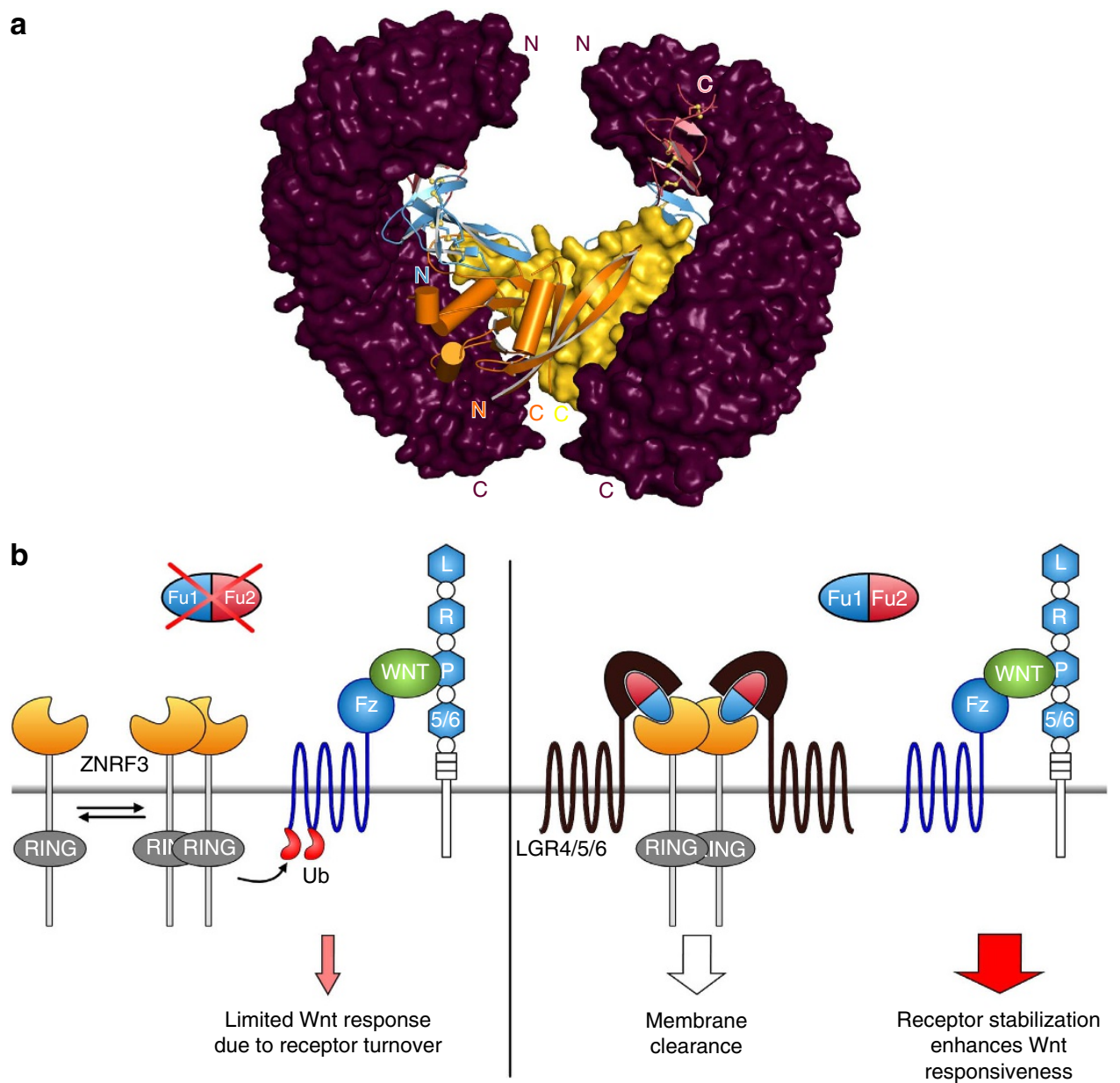

Figure 7 | Modelling of a ternary 2:2:2 LGR $_{\text {ecto }}-\mathbf{R s p o}_{\mathrm{Fu} 1-F u 2}-\mathbf{Z N R F}_{\text {ecto }}$ complex and its implication for signalling. (a) The $\mathrm{hLGR5}$ ecto $\times$ hRSPO1 ${ }_{\mathrm{Fu} 1-\mathrm{Fu} 2} \times$ mZNRF3 $3_{\text {ecto }}$ 2:2:2 complex was generated by superposing the ternary hLGR5 ecto $\times$ RSPO1 Fu1-Fu2 $\times$ hRNF43 ecto $_{\text {complex }}{ }^{25}$ (Protein Data Bank ID code

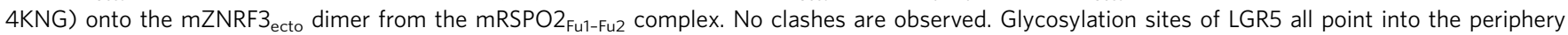
of the shown complex. (b) A model for regulation of Wnt signalling by RSPO and its receptors based on our results and those by Hao et al. ${ }^{16}$ The schematic model takes into account the different binding sites of LGRs and ZNRF3/RNF43 on Rspos as determined by us and others $25-28$.

$16.2 \mathrm{mg} \mathrm{ml}^{-1}$ in $0.5 \mathrm{M} \mathrm{Li}_{2} \mathrm{SO}_{4}$ and $10 \%$ (w/v) PEG8000 (form IV). The S90C variant of mZNRF3 $3_{\text {ecto }}$ crystallized in $45 \%(\mathrm{v} / \mathrm{v}) 2$-methyl-2,4-pentanediol, $200 \mathrm{mM}$ ammonium acetate and $100 \mathrm{mM}$ Bis-Tris, $\mathrm{pH} 5.5$, at a concentration of $38.5 \mathrm{mg} \mathrm{ml}^{-1}$. Apo xZNRF3 ecto crystallized at $25 \mathrm{mg} \mathrm{ml}^{-1}$ in $20 \%(\mathrm{w} / \mathrm{v})$ PEG3350, $100 \mathrm{mM}$ Bis-Tris propane, $\mathrm{pH} 6.5,200 \mathrm{mM} \mathrm{NaBr}$ (form I), and 20\% (w/v) PEG3350 and $0.200 \mathrm{M} \mathrm{NaCl}$ (form II). Crystals composed of the complex of $\mathrm{mZNRF} 3_{\text {ecto }}$ and $\mathrm{mRSPO} 2_{\mathrm{Fu} 1-\mathrm{Fu} 2}$ were obtained at a concentration of $18 \mathrm{mg} \mathrm{ml}^{-1}$ in $1.8 \mathrm{M}$ AS, $100 \mathrm{mM}$ Bis-Tris, $\mathrm{pH} 6.5,2 \%$ (v/v) PEGMME550. Crystals of the mixed species complexes of $\mathrm{mZNRF} 3_{\text {ecto }}$ and SeMet $\mathrm{xRSPO} 2_{\mathrm{Fu} 1-\mathrm{Fu} 2}$ appeared in 25\% (w/v) PEG4000, $200 \mathrm{mM} \mathrm{NaCl}, 100 \mathrm{mM}$ HEPES/NaOH, pH 7.5 (form I), and $20 \%(\mathrm{w} / \mathrm{v})$ PEG3350, $200 \mathrm{mM}$ sodium citrate, $100 \mathrm{mM}$ Bis-Tris propane, $\mathrm{pH} 6.5$ (form II). xZNRF3 $3_{\text {ecto }}-\mathrm{xRSPO} 2_{\mathrm{Fu} 1-\mathrm{Fu} 2}$ complexes crystallized in $20 \%(\mathrm{w} / \mathrm{v})$ PEG3350, $200 \mathrm{mM}\left(\mathrm{NH}_{4}\right) \mathrm{F}$ (form I) and 20\% (w/v) PEG6000, $100 \mathrm{mM} \mathrm{MES,} \mathrm{pH}$ 6.0 (form II). The complex of $x R N F 43_{\text {ecto }}$ and $x R S P O 2_{\mathrm{Fu} 1-\mathrm{Fu} 2}$ was crystallized in condition A1 of the PACT premier screen from Molecular Dimensions. For cryoprotection, crystals were transferred to mother liquor supplemented with $1.7 \mathrm{M}$ sodium malonate, $\mathrm{pH} 7$ (both apo xRSPO2 ${ }_{\mathrm{Fu} 1-\mathrm{Fu} 2}$ crystals), with AS to $3 \mathrm{M}$ (mouse/ mouse complex), or with PEG200 to achieve total (PEG, polyethylene glycol) $>30 \%$ (all other ZNRF3 apo and complex crystals) by incrementally adjusting the concentration of the cryoprotectant. Crystal were then flash-cooled by dipping into liquid nitrogen. The $\mathrm{xRNF} 43_{\text {ecto }}-\mathrm{xRSPO} 2_{\mathrm{Fu} 1-\mathrm{Fu} 2}$ complex crystal was frozen directly and showed strong ice rings. Diffraction data were collected at DIAMOND synchrotron light source at the beamlines i02, i03, i04 and i24. Crystal

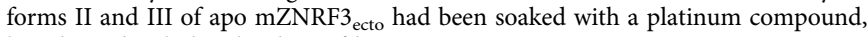
but showed only low binding of heavy atoms.

Structure determination. The structure of xRspo2 $2_{\mathrm{Fu} 1-\mathrm{Fu} 2}$ was solved using highly redundant single-wavelength anomalous dispersion (SAD) data from a $\mathrm{Pt}(\mathrm{IV})$ - soaked crystal that diffracted to $3.2 \AA$ (Table 1). Ten strong anomalous sites could be identified by AUTOSHARP ${ }^{33}$. Refinement and subsequent density modification with SOLOMON lead to clearly interpretable electron density. A partial model obtained from BUCCANEER ${ }^{34}$ was used to solve the high-resolution structure. The model was improved with iterative rounds of manual building in $\mathrm{COOT}^{35}$ and refinement in REFMAC5 (ref. 36). The structure of mZNRF3 $3_{\text {ecto }}$ in complex with SeMet-labelled xRSPO2 ${ }_{\mathrm{Fu} 1-\mathrm{Fu} 2}$ was solved from SAD data collected at the Se $\mathrm{K}$-absorption edge. Albeit only one component of the complex was labelled and the complex being crystallized in the low-symmetry space group P1, the Se atom substructure (four sites) could be identified by PHENIX HYSS ${ }^{37}$ from average redundancy data (Supplementary Table S1). An initial model generated by AUTOSOL was used to solve the high-resolution $\mathrm{mZNRF} 3_{\text {ecto }}$ structure (Supplementary Table S1). All other structures were solved by molecular replacement with PHASER ${ }^{38}$ and completed by manual rebuilding in COOT and refinement with REFMAC5. Models were validated with MOLPROBITY ${ }^{39}$. Superpositions were performed within CCP4 or COOT using the SSM algorithm. Electrostatics potentials were generated using $\mathrm{APBS}^{40}$, surface sequence conservation was calculated using CONSURF ${ }^{41}$ and interface areas of proteins were calculated using the PISA web server ${ }^{42}$. Figures were produced in PYMOL and assembled in PHOTOLINE32.

Analytical ultracentrifugation. $\mathrm{xZNRF} 3_{\text {ecto }}-\mathrm{xRSPO} 2_{\mathrm{Fu} 1-\mathrm{Fu} 2}$ and $\mathrm{xRNF} 43_{\text {ecto }}-$ $\mathrm{xRSPO}_{\mathrm{Fu} 1-\mathrm{Fu} 2}$ complexes and apo $\mathrm{mZNRF}_{\text {ecto }}$ variants at $350 \mu \mathrm{M}$ in $10 \mathrm{mM}$ HEPES/NaOH, $250 \mathrm{mM} \mathrm{NaCl}$ were subjected to sedimentation velocity experiments at $20^{\circ} \mathrm{C}$ using an Optima Xl-I analytical ultracentrifuge (Beckman) with $3 \mathrm{~mm}$ or $12 \mathrm{~mm}$ double sector centerpieces in an An-60 Ti rotor (Beckman) at 40,000 r.p.m. Sedimentation was monitored by ultraviolet absorption at $300 \mathrm{~nm}$ and by Rayleigh interference. Data were analysed using SEDFIT operating in $c(s)$ and $c\left(s, f / f_{\mathrm{o}}\right)$ modes (with a frictional coefficient range of $1-2$ in the latter case and a 
resolution in s of 100 $)^{43}$. The resulting sedimentation coefficient distributions were plotted using ProFit (Uetikon am See, $\mathrm{CH}$ ). The crystal structures were modelled hydrodynamically using the programme HYDROPRO ${ }^{44}$

SPR equilibrium binding studies. Affinity between variants of $\mathrm{mZNRF} 3_{\text {ecto }}$, human RNF43 ${ }_{\text {ecto }}$ and $\mathrm{mRSPO} 2_{\mathrm{Fu} 1-\mathrm{Fu} 2}$ was measured at $25^{\circ} \mathrm{C}$ in $10 \mathrm{mM}$ HEPES/ $\mathrm{NaOH}, \mathrm{pH}$ 7.5, $150 \mathrm{mM} \mathrm{NaCl}, 0.005 \%$ Tween 20 using a Biacore T200 machine (GE Healthcare). Synthetic DNA corresponding to mZNRF3(K53-L205), hRNF43(Q44L188) and mRSPO2(I39-G144), as well as variants thereof, was obtained from Invitrogen/Geneart (Germany) and cloned into a variant of the pHLsec vector encoding a C-terminal recognition sequence for the Escherichia coli BirA enzyme. Biotinylation at this sequence tag was performed as described ${ }^{45}$. Experiments were performed as described before ${ }^{46}$, with the biotinylated variants immobilized to the chip surface precoupled with approximately 10,000 resonance units (RU) of streptavidin. Immobilized protein amounts varied between 350 and 1,000 RU (1 experiment with 1,650 RU). The amount of immobilized protein did not seem to strongly influence the binding model. After each injection of analyte, the chip surface was regenerated with $2 \mathrm{M} \mathrm{MgCl}_{2}, 10 \mathrm{mM}$ HEPES/NaOH, pH 7.5 (RSPO coupled), $100 \mathrm{mM}$ phosphate, $\mathrm{pH} 3.7,2 \mathrm{M} \mathrm{NaCl}$ and $1 \%(\mathrm{v} / \mathrm{v})$ Tween20 (RNF43 or ZNRF3 coupled) or $25 \%$ ethylene glycol, $2 \mathrm{M} \mathrm{NaCl}, 100 \mathrm{mM} \mathrm{HEPES} / \mathrm{NaOH}, \mathrm{pH}$ $7.5,1 \%$ Tween20 (LGR coupled) to return to baseline levels. Data were fitted to a Langmuir adsorption model $B=B_{\max } C /\left(K_{\mathrm{d}}+C\right)$, where $B$ is the amount of bound analyte and $C$ is the concentration of analyte in the sample. Data were then normalized to a maximum analyte-binding value of 100 . Unless stated otherwise, data points correspond to average from two independent dilution series.

Co-immunoprecipitation binding assays. Full-length human RSPO1-Myc (1-263), and domains of Fu1/Fu2 (1-147) and TSR (1-20, 144-263), were originally reported in ref. 47 . On the basis of the disulphide bond pattern resolved in the crystal structure, new individual $\mathrm{Fu}$ domain deletions were generated: deltaFurin1 (del 39-94; Rspo1 ${ }_{\Delta \mathrm{Fu} 1}$ ) and deltaFurin2 (del 97-142; Rspo1 ${ }_{\Delta \mathrm{Fu} 2}$ ). For immunoprecipitation, conditioned medium from HEK293T cells transfected using FugeneHD with RSPO1-Myc, Furin1/2-Myc, TSR-Myc, deltaFurin1-Myc or deltaFurin2-Myc was mixed with conditioned medium from cells transfected with IgG, Human RNF43 ECD-IgG (1-198) or mouse HA-Lgr4 ECD (from ref. 11), and incubated at $4{ }^{\circ} \mathrm{C}$ overnight. The mixture was then incubated with protein G-agarose beads for $2 \mathrm{~h}$ at $4{ }^{\circ} \mathrm{C}$ and washed with buffer $(150 \mathrm{mM} \mathrm{NaCl}, 1 \mathrm{mM}$ EDTA, $2.5 \mathrm{mM}$ EGTA, $10 \%$ glycerol, $0.1 \%$ Tween 20 with protease inhibitors). Protein was eluted using $2 \times$ SDS sample buffer and separated by SDS-PAGE. Western blotting was performed by using horseradish peroxidase-conjugated antihuman IgG (Calbiochem), anti-HA or anti-c-Myc. For co-immunoprecipitation assays, anti-Myc (9E10, Santa Cruz) was used at a 1:100 concentration. For western blots, primary antibodies were diluted 1:1000 and secondary antibodies were diluted 1:10000 from stocks.

Activity assays. To assess Rspo activation of the Wnt signalling pathway, a traditional dual-luciferase assay consisting of the Wnt-responsive SuperTopFlash reporter (normalized to a control promoter driving Renilla luciferase) was used as previously described ${ }^{46}$. Mammalian cell transfections were done in HEK293T (ATCC CRL-11268) cells and performed in triplicate for each sample condition. Cells were plated at $1 \times 10^{5}$ per $\mathrm{ml}$ in 24 -well plates and transfected the following day with a total of $200 \mathrm{ng}$ of DNA per well (50 ng SuperTopFlash, $10 \mathrm{ng}$ TK-Renilla, experimental expression vectors and balanced with empty vector). Lysates were collected $36 \mathrm{~h}$ post transfection and used with the Dual-luciferase reporter system (Promega). Firefly and Renilla luciferase activity was measured using the Wallac 1420 multilabel counter in 96-well plates. Normalized data expressed in relative luciferase units was averaged from triplicate assays and error bars reflect s.d. Representative results are shown from one of multiple independent experiments.

\section{References}

1. Kazanskaya, O. et al. R-Spondin2 is a secreted activator of Wnt/beta-catenin signaling and is required for Xenopus myogenesis. Dev. Cell 7, 525-534 (2004).

2. Kim, K. A. et al. Mitogenic influence of human R-spondin1 on the intestinal epithelium. Science 309, 1256-1259 (2005).

3. de Lau, W. B., Snel, B. \& Clevers, H. C. The R-spondin protein family. Genome Biol. 13, 242 (2012).

4. Parma, P. et al. R-spondin1 is essential in sex determination, skin differentiation and malignancy. Nat. Genet. 38, 1304-1309 (2006).

5. Zhao, J. et al. R-spondin1, a novel intestinotrophic mitogen, ameliorates experimental colitis in mice. Gastroenterology 132, 1331-1343 (2007).

6. Papapietro, O. et al. R-Spondin 2 signalling mediates susceptibility to fatal infectious diarrhoea. Nat. Commun. 4, 1898 (2013).

7. Seshagiri, S. et al. Recurrent R-spondin fusions in colon cancer. Nature 488 , 660-664 (2012).

8. Blaydon, D. C. et al. The gene encoding R-spondin 4 (RSPO4), a secreted protein implicated in Wnt signaling, is mutated in inherited anonychia. Nat. Genet. 38, 1245-1247 (2006).
9. Chishti, M. S., Kausar, N., Rafiq, M. A., Amin, M. \& Ahmad, W. A novel missense mutation in RSPO4 gene underlies autosomal recessive congenital anonychia in a consanguineous Pakistani family. Br. J. Dermatol. 158, 621-623 (2008).

10. Khan, T. N. et al. Novel missense mutation in the RSPO4 gene in congenital hyponychia and evidence for a polymorphic initiation codon (p.M1I). BMC Med. Genet. 13, 120 (2012).

11. Carmon, K. S., Gong, X., Lin, Q., Thomas, A. \& Liu, Q. R-spondins function as ligands of the orphan receptors LGR4 and LGR5 to regulate Wnt/beta-catenin signaling. Proc. Natl Acad. Sci. USA 108, 11452-11457 (2011).

12. Carmon, K. S., Lin, Q., Gong, X., Thomas, A. \& Liu, Q. LGR5 interacts and cointernalizes with Wnt receptors to modulate Wnt/beta-catenin signaling. Mol. Cell Biol. 32, 2054-2064 (2012).

13. de Lau, W. et al. Lgr5 homologues associate with Wnt receptors and mediate R-spondin signalling. Nature 476, 293-297 (2011).

14. Glinka, A. et al. LGR4 and LGR5 are R-spondin receptors mediating Wnt/betacatenin and Wnt/PCP signalling. EMBO Rep. 12, 1055-1061 (2011).

15. Ruffner, H. et al. R-Spondin potentiates Wnt/beta-catenin signaling through orphan receptors LGR4 and LGR5. PLoS One 7, e40976 (2012).

16. Hao, H. X. et al. ZNRF3 promotes Wnt receptor turnover in an R-spondinsensitive manner. Nature 485, 195-200 (2012).

17. Wu, J. et al. Whole-exome sequencing of neoplastic cysts of the pancreas reveals recurrent mutations in components of ubiquitin-dependent pathways. Proc. Natl Acad. Sci. USA 108, 21188-21193 (2011).

18. Koo, B. K. et al. Tumour suppressor RNF43 is a stem-cell E3 ligase that induces endocytosis of Wnt receptors. Nature 488, 665-669 (2012).

19. Kim, K. A. et al. R-Spondin family members regulate the Wnt pathway by a common mechanism. Mol. Biol. Cell 19, 2588-2596 (2008).

20. Nam, J. S., Turcotte, T. J., Smith, P. F., Choi, S. \& Yoon, J. K. Mouse cristin/R spondin family proteins are novel ligands for the Frizzled 8 and LRP6 receptors and activate beta-catenin-dependent gene expression. J. Biol. Chem. 281, 13247-13257 (2006).

21. Li, S. J. et al. Loss-of-function point mutations and two-furin domain derivatives provide insights about R-spondin 2 structure and function. Cell Signal. 21, 916-925 (2009).

22. Mahon, P. \& Bateman, A. The PA domain: a protease-associated domain Protein Sci. 9, 1930-1934 (2000).

23. Anandasabapathy, N. et al. GRAIL: an E3 ubiquitin ligase that inhibits cytokine gene transcription is expressed in anergic CD4 $+\mathrm{T}$ cells. Immunity 18, 535-547 (2003).

24. Nurieva, R. I. et al. The E3 ubiquitin ligase GRAIL regulates T cell tolerance and regulatory $\mathrm{T}$ cell function by mediating $\mathrm{T}$ cell receptor-CD3 degradation. Immunity 32, 670-680 (2010).

25. Chen, P. H., Chen, X., Lin, Z., Fang, D. \& He, X. The structural basis of R-spondin recognition by LGR5 and RNF43. Genes Dev. 27, 1345-1350 (2013).

26. Peng, W. C. et al. Structure of stem cell growth factor R-spondin 1 in complex with the ectodomain of its receptor LGR5. Cell Rep. 3, 1885-1892 (2013).

27. Wang, D. et al. Structural basis for R-spondin recognition by LGR4/5/6 receptors. Genes Dev. 27, 1339-1344 (2013).

28. Xu, K., Xu, Y., Rajashankar, K. R., Robev, D. \& Nikolov, D. B. Crystal structures of Lgr4 and its complex with R-spondin1. Structure 21, 1683-1689 (2013).

29. Deshaies, R. J. \& Joazeiro, C. A. RING domain E3 ubiquitin ligases. Annu. Rev. Biochem. 78, 399-434 (2009).

30. Plechanovova, A. et al. Mechanism of ubiquitylation by dimeric RING ligase RNF4. Nat. Struct. Mol. Biol. 18, 1052-1059 (2011).

31. Aricescu, A. R., Lu, W. \& Jones, E. Y. A time- and cost-efficient system for high-level protein production in mammalian cells. Acta Crystallogr. D Biol. Crystallogr. 62, 1243-1250 (2006).

32. Walter, T. S. et al. A procedure for setting up high-throughput nanolitre crystallization experiments. Crystallization workflow for initial screening, automated storage, imaging and optimization. Acta Crystallogr. D Biol. Crystallogr. 61, 651-657 (2005).

33. Vonrhein, C., Blanc, E., Roversi, P. \& Bricogne, G. Automated structure solution with autoSHARP. Methods Mol. Biol. 364, 215-230 (2007).

34. Cowtan, K. The Buccaneer software for automated model building. 1. Tracing protein chains. Acta Crystallogr. D Biol. Crystallogr. 62, 1002-1011 (2006).

35. Emsley, P. \& Cowtan, K. Coot: model-building tools for molecular graphics. Acta Crystallogr. D. Biol. Crystallogr. 60, 2126-2132 (2004).

36. Murshudov, G. N., Vagin, A. A. \& Dodson, E. J. Refinement of macromolecular structures by the maximum-likelihood method. Acta Crystallogr. D Biol. Crystallogr. 53, 240-255 (1997).

37. Adams, P. D. et al. PHENIX: a comprehensive Python-based system for macromolecular structure solution. Acta Crystallogr. D Biol. Crystallogr. 66, 213-221 (2010).

38. McCoy, A. J. et al. Phaser crystallographic software. J. Appl. Crystallogr. 40, 658-674 (2007). 
39. Davis, I. W. et al. MolProbity: all-atom contacts and structure validation for proteins and nucleic acids. Nucleic Acids Res. 35, W375-W383 (2007).

40. Baker, N. A., Sept, D., Joseph, S., Holst, M. J. \& McCammon, J. A. Electrostatics of nanosystems: application to microtubules and the ribosome. Proc. Natl Acad. Sci. USA 98, 10037-10041 (2001).

41. Ashkenazy, H., Erez, E., Martz, E., Pupko, T. \& Ben-Tal, N. ConSurf 2010 calculating evolutionary conservation in sequence and structure of proteins and nucleic acids. Nucleic Acids Res. 38, W529-W533 (2010).

42. Krissinel, E. \& Henrick, K. Inference of macromolecular assemblies from crystalline state. J. Mol. Biol. 372, 774-797 (2007).

43. Schuck, P. Size-distribution analysis of macromolecules by sedimentation velocity ultracentrifugation and lamm equation modeling. Biophys. J 78, 1606-1619 (2000).

44. Ortega, A., Amoros, D. \& T. J., G. Prediction of hydrodynamic and other solution properties of rigid proteins from atomic- and residue-level models. Biophys. J. 101, 892-898 (2011).

45. O'Callaghan, C. A. et al. BirA enzyme: production and application in the study of membrane receptor-ligand interactions by site-specific biotinylation. Anal. Biochem. 266, 9-15 (1999).

46. Chen, S. et al. Structural and functional studies of LRP6 ectodomain reveal a platform for Wnt signaling. Dev. Cell 21, 848-861 (2011).

47. Wei, Q. et al. R-spondin1 is a high affinity ligand for LRP6 and induces LRP6 phosphorylation and beta-catenin signaling. J. Biol. Chem. 282, 15903-15911 (2007).

48. Ayadi, L. Molecular modelling of the TSR domain of R-spondin 4. Bioinformation 3, 119-123 (2008).

\section{Acknowledgements}

We thank Diamond Light Source for beamtime (proposal mx8423) and the staff of beamlines I02, I03, I04, I04-1 and I24 for assistance with crystal testing and data collection. We thank W. Lu and Y. Zhao for help with tissue culture, R. Chen for contributions to protein production, K. Harlos and T. Walter for assistance with crystallization and T. Malinauskas for discussion. The analytical ultracentrifugation experiments were performed in the Biophysical Facility at the Department of Biochemistry, University of Oxford. X.H. acknowledges F. Cong (Novartis) for human ZNRF3 and RNF43 constructs. This work was funded by Cancer Research UK, the UK Medical Research Council (to E.Y.J., A10976 and G9900061) and NIH (to X.H., RO1-
GM057603 and GM057603S1). M.Z. holds an IEF Marie Curie fellowship, C.K. is the recipient of a Wellcome Trust D. Phil. studentship and Y.X. is supported in part by a graduate studentship from the Chinese Scholarship Council. The Wellcome Trust Centre for Human Genetics is supported by Wellcome Trust grant 090532/Z/09/Z. X.H. is an endowed research chair of Boston Children's Hospital (BCH) and acknowledges support by BCH Intellectual and Developmental Disabilities Research Center (P30 HD-18655).

\section{Author contributions}

All authors contributed to the design of the project, data analysis and preparation of the manuscript. M.Z. cloned, purified and performed SPR and AUC experiments on Rspo proteins and ZNRF3, crystallized and solved the individual and complex structures. C.K. contributed to cloning, protein purification, SPR and diffraction data collection. R.J.C.G. contributed to AUC data collection and analysis. B.T.M., Y.X. and M.C. cloned and performed functional assays and co-immunoprecipitation for Rspo proteins.

\section{Additional information}

Accession codes: Coordinates and structure factors for $z Z N R F 3_{\text {ecto }}, \mathrm{mZNRF}_{\text {ecto }}$ $\mathrm{xZNRF}_{\text {ecto }}, \mathrm{xRspo}_{\mathrm{Fu} 1-\mathrm{Fu} 2}, \mathrm{mZNRF} 3_{\text {ecto }}-\mathrm{mRspo} 2_{\mathrm{Fu} 1-\mathrm{Fu} 2}, \mathrm{mZNRF} 3_{\text {ecto }}-\mathrm{xRspo} 2_{\mathrm{Fu} 1-\mathrm{Fu}}$, $\mathrm{xZNRF} 3_{\text {ecto }}-\mathrm{xRspo} 2_{\mathrm{v}}$ and $\mathrm{xRNF} 43_{\text {ecto }}-\mathrm{xRspo} 2_{\mathrm{Fu} 1-\mathrm{Fu} 2}$ crystal structures have been deposited in the Protein Data Bank with the succession numbers 4C84, 4C85, 4C86, 4C8A, 4C8C, 4C8F, 4C8P, 4C8T, 4C8U, 4C8V, 4C8W, 4C99, 4C9A, 4C9E, 4C9R, 4C9U and $4 \mathrm{C} 9 \mathrm{~V}$ as also given in Table 1 and Supplementary Table S1.

Supplementary Information accompanies this paper at http://www.nature.com/ naturecommunications

Competing financial interests: The authors declare no competing financial interests.

Reprints and permission information is available online at http://npg.nature.com/ reprintsandpermissions/

How to cite this article: Zebisch, M. et al. Structural and molecular basis of ZNRF3/ RNF43 transmembrane ubiquitin ligase inhibition by the Wnt agonist R-spondin. Nat. Commun. 4:2787 doi: 10.1038/ncomms3787 (2013).

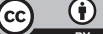

This article is licensed under a Creative Commons Attribution 3.0 Unported Licence. To view a copy of this licence visit http:// creativecommons.org/licenses/by/3.0/ 